\title{
Reasonably Programmable Literal Notation
}

\author{
CYRUS OMAR*, University of Chicago, USA \\ JONATHAN ALDRICH, Carnegie Mellon University, USA
}

General-purpose programming languages typically define literal notation for only a small number of common data structures, like lists. This is unsatisfying because there are many other data structures for which literal notation might be useful, e.g. finite maps, regular expressions, HTML elements, SQL queries, syntax trees for various languages and chemical structures. There may also be different implementations of each of these data structures behind a common interface that could all benefit from common literal notation. This paper introduces typed literal macros (TLMs), which allow library providers to define new literal notation of nearly arbitrary design at any specified type or parameterized family of types. Compared to existing approaches, TLMs are uniquely reasonable. TLM clients can reason abstractly, i.e. without examining grammars or generated expansions, about types and binding. The system only needs to convey to clients, via secondary notation, the inferred segmentation of each literal body, which gives the locations and types of spliced subterms. TLM providers can reason modularly about syntactic ambiguity and expansion correctness according to clear criteria This paper incorporates TLMs into Reason, an emerging alternative front-end for OCaml, and demonstrates, through several non-trivial case studies, how TLMs integrate with the advanced features of OCaml, including pattern matching and the module system. We also discuss optional integration with MetaOCaml, which allows TLM providers to be more confident about type correctness. Finally, we establish these abstract reasoning principles formally with a detailed type-theoretic account of expression and pattern TLMs for "core ML".

\section{CCS Concepts: • Software and its engineering $\rightarrow$ Extensible languages; Macro languages;}

Additional Key Words and Phrases: parsing, macro systems, hygiene, type systems, pattern matching

ACM Reference Format:

Cyrus Omar and Jonathan Aldrich. 2018. Reasonably Programmable Literal Notation. Proc. ACM Program. Lang. 2, ICFP, Article 106 (September 2018), 32 pages. https://doi.org/10.1145/3236801

\section{INTRODUCTION}

When designing the surface syntax of a general-purpose programming language, it is common practice to define shorthand literal notation, i.e. notation that decreases the syntactic cost of constructing and pattern matching over values of some particular data structure or parameterized family of data structures. For example, many languages in the ML family support list literals like [x1, x2, x3] in both expression and pattern position [Harper 1997; Milner et al. 1997]. Lists are common across problem domains, but other literal notation is more specialized. For instance, Ur/Web extends the surface syntax of Ur (an ML-like language [Chlipala 2010]) with expression and pattern literals for encodings of XML and HTML data [Chlipala 2015]. For example, Fig. 1 shows two Ur/Web HTML literals, one that "splices in" a string expression delimited by $\{[$ and $]\}$ and the other an HTML expression delimited by $\{$ and $\}$.

\footnotetext{
*The majority of this research was performed while the first author attended Carnegie Mellon University.
}

Authors' addresses: Cyrus Omar, University of Chicago, Chicago, IL, USA, comar@cs.uchicago.edu; Jonathan Aldrich, Carnegie Mellon University, Pittsburgh, PA, USA, jonathan.aldrich@cs.cmu.edu.

This work is licensed under a Creative Commons Attribution 4.0 International License.

(C) 2018 Copyright held by the owner/author(s).

2475-1421/2018/9-ART106

https://doi.org/10.1145/3236801

Proc. ACM Program. Lang., Vol. 2, No. ICFP, Article 106. Publication date: September 2018. 
fun heading first_name $=\langle x m l\rangle\langle h 1\rangle$ Hello, $\{[$ first_name $]\} !\langle/ h 1\rangle\langle/ x m l\rangle$

val body $=\langle x m l><$ body $>\{$ heading "World" $\} \ldots</$ body $\rangle\langle/ x m l\rangle$

Fig. 1. HTML literals with support for splicing at two types are built primitively into Ur/Web [Chlipala 2015].

This design practice, where the language designer privileges certain library constructs with built-in literal notation, is ad hoc in that it is easy to come up with other examples of data structures for which mathematicians, scientists or programmers have invented specialized notation [Cajori 1928; Iverson 1980; Omar et al. 2014]. For example, (1) clients of a "collections" library might want not just list literals, but also literal notation for matrices, finite maps, and so on; (2) clients of a "web programming" library might want CSS literals (which Ur/Web lacks); (3) a compiler author might want "quotation" literals for the terms of the object language and various intermediate languages of interest; and (4) clients of a "chemistry" library might want chemical structure literals based on the SMILES standard [Anderson et al. 1987].

Although requests for specialized literal notation are easy to dismiss as superficial, the reality is that literal notation, or the absence thereof, can have a substantial influence on software quality. For example, Bravenboer et al. [2007] finds that literal notation for structured encodings of queries, like the SQL-based query literals now found in many languages [Meijer et al. 2006], reduce the temptation to use string encodings of queries and therefore reduce the risk of catastrophic string injection attacks [OWASP 2017]. More generally, evidence suggests that programmers frequently resort to "stringly-typed programming", i.e. they choose strings instead of composite data structures, largely for reasons of notational convenience. In particular, Omar et al. [2014] sampled strings from open source projects and found that at least $15 \%$ of them could be parsed by some readily apparent type-specific grammar, e.g. for URLs, paths, regular expressions and many others. Literal notation, with support for splicing, would decrease the syntactic cost of composite encodings, which are more amenable to programmatic manipulation and compositional reasoning than string encodings.

Of course, it would not scale to ask general-purpose language designers to build in support for all known notations a priori. Instead, there has been persistent interest in mechanisms that allow library providers to define new literal notation on their own. For example, direct grammar extension systems like Camlp4 [de Rauglaudre 2003] and Sugar* [Erdweg et al. 2011; Erdweg and Rieger 2013], term rewriting systems like Template Haskell [Mainland 2007; Sheard and Peyton Jones 2002], the system of type-specific languages (TSLs) described by Omar et al. [2014], and other systems that we will discuss below can all be used to define new literal notation (and, in some cases, other forms of new notation, such as new infix operator forms, control flow operations or type declaration forms, which we leave beyond the scope of this paper).

Problem The problem that specifically motivates this paper is that these existing systems make it difficult or impossible to reason abstractly about such fundamental issues as types and variable binding when presented with a program using user-defined literal forms. Instead, programmers and editor services can only reason transparently, i.e. by inspecting the underlying expansion or the implementation details of the collection of extensions responsible for producing the expansion.

Consider, for instance, the perspective of a programmer trying to comprehend the program text in Fig. 2a, which is written in an emerging dialect of OCaml's surface syntax called Reason [Reason Team 2018] that has, hypothetically, been extended with some number of new literal forms by a grammar extension system - Lines 1-6 outline the Camlp4 mechanism [de Rauglaudre 2003]; Sugar*/SugarHaskell is similar [Erdweg et al. 2011, 2012]. Line 8 uses one of these active syntax extensions to construct an encoding of a query in the rather obscure database query language $\mathrm{K}$, using its intentionally terse notation [Whitney and Shasha 2001]. The problem is that a programmer examining the program as presented, and unfamiliar with (i.e. holding abstract) the details elided on Lines 1-6, cannot easily answer questions like the following: 


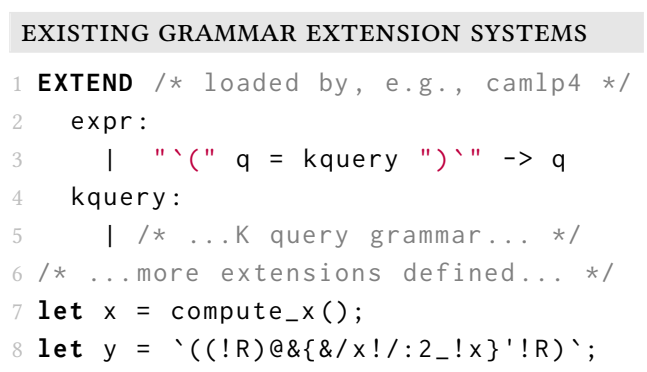

(a) It is difficult to reason abstractly given program text that uses a variety of grammar extensions (see the six reasoning criteria in the paper text).

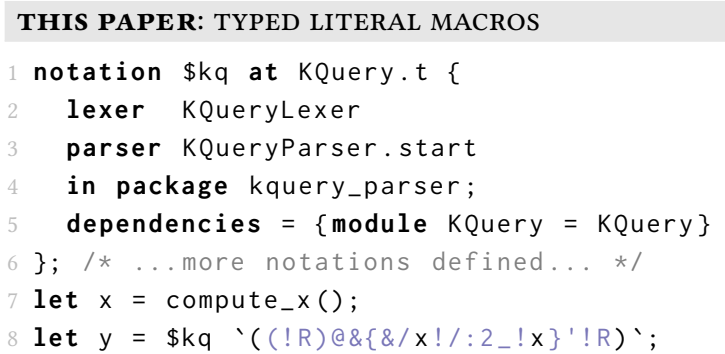

(b) TLMs make examples like these more reasonable by leaving the base grammar fixed and strictly enforcing a simple type, binding and segmentation discipline.

Fig. 2. Two of the possible ways to introduce literal notation for encodings of $\mathrm{K}$ queries

(1) Responsibility: Which syntax extension determined the expansion of the literal on Line 8? Might activating a new extension generate a conflicting expansion for the same literal?

(2) Expansion Typing: What type does the expansion, and thus the variable y on Line 8, have?

(3) Context Dependence: Which bindings does the expansion of Line 8 invisibly depend on? If we shadow or remove a module or other binding, could that break or change the meaning of Line 8 because its expansion depends invisibly on the original binding?

(4) Segmentation: Are the characters $x, R$ and 2 on Line 8 parsed as spliced expressions, meaning that they appear directly in the underlying expansion, or are they parsed in some other way peculiar to this literal notation, e.g. as operators in the K query language?

(5) Segment Typing: What type is each spliced term expected to have? How can we infer a type for a variable that appears in a spliced term without looking at where it ends up in the expansion?

(6) Capture: If $x$ is in fact a spliced term, does it refer to the binding of $x$ on Line 7, or might it capture an invisible binding of the same identifier in the expansion of Line 8 ?

Forcing the programmer to reason transparently to answer basic questions like these defeats the ultimate purpose of syntactic sugar: decreasing cognitive cost [Green 1989]. analogous problems do not arise when programming without syntax extensions in languages like ML - programmers can reason lexically about where variables and other symbols are bound, and types mediate abstraction over function and module implementations [Reynolds 1983]. Ideally, the programmer would be able to abstract in some analogous manner over the implementation of an unfamiliar notation.

Given these issues, we concluded that direct grammar extension systems like camlp4 were not ideally suited for integration into the Reason platform, which seeks to develop a clear and modern surface syntax for the OCaml programming language [Reason Team 2018]. We also evaluated various approaches that are based not on direct grammar extension but on term rewriting over a fixed grammar. We give a full account of this evaluation in Sec. 7, but briefly, we found that:

- Unhygienic approaches like OCaml's preprocessor extension point (PPX) rewriters [Leroy et al. 2014] and Template Haskell [Mainland 2007; Sheard and Peyton Jones 2002] allow us to define new literal notation with support for splicing by repurposing existing string literal forms. They also partially or completely solve the problem of reasoning about Responsibility but they do not satisfy the remaining five reasoning criteria.

- Hygienic term rewriting macro systems, like those in various Lisp-family languages, e.g. Racket [Flatt 2012], as well as Scala [Burmako 2013], do not allow us to flexibly repurpose string literal forms to define composite literal forms because the hygiene discipline cannot account for base language terms spliced out of string literal bodies via parsing (see Sec. 7 for more details). 
- Type-specific languages (TSLs) [Omar et al. 2014] come closer to our goals in that they explicitly support splicing terms out of literal bodies, but the mechanism falls subtly short with regard to the six reasoning principles just discussed in ways that we detail in Sec. 7. In any case, this approach was designed for simple nominally-typed languages and relies critically on a particular local type inference scheme. It is not immediately suitable for a language with an ML-like semantics, meaning a language with support for structural types (like tuple and function types in ML), parameterized type families, pattern matching and non-local type inference.

Contributions This paper introduces typed literal macros (TLMs): the first system for defining new literal notation that (1) provides the ability to reason abstractly about all six of the topics just outlined; and (2) is semantically expressive enough for integration into Reason/OCaml and other full-scale statically typed functional languages. We evaluate these claims with a number of non-trivial examples appearing throughout the paper that involve the advanced language features mentioned above. In describing these examples, we demonstrate that literal parsing logic can be defined using standard, unmodified parser generators, so the burden on notation providers is comparable to that of existing systems despite these stronger reasoning principles. Finally, we give a type-theoretic account of TLMs where we formally establish these abstract reasoning principles.

A Brief Overview For a brief overview of the proposed mechanism, consider Fig. 2b. Lines 1-6 define a TLM named $\$ \mathrm{kq}$ that provides K query literal notation. Line 8 applies this TLM to express the example from Fig. 2a. We can reason abstractly about this program as follows.

(1) Responsibility: The lexer and parser specified by the applied TLM on Lines 2-4 are together exclusively responsible for lexing, parsing and expanding the body of the generalized literal form, i.e. the characters between '( and )'. We will give more details on generalized literal forms and on constructing a TLM lexer and parser in the next section. For now, let us simply reiterate that our design goal is to provide a system where the programmer does not normally need to look up the definitions of KQueryLexer and KQueryParser to reason about types and binding.

(2) Expansion Typing: The type annotation on Line 1 specifies the type that every expansion generated by \$kq must have, here KQuery.t.

(3) Context Dependence: Line 5 specifies that expansions generated by \$kq are allowed to use the module KQuery, and no others. The system ensures that this dependency is bound as specified even if the variable KQuery has been shadowed at the application site. This completely relieves clients from needing to consider expansion-internal dependencies when naming variables.

(4) Segmentation: The intermediate output that the TLM generates is structured so that the system can infer from it an accurate segmentation of the literal body that distinguishes spliced terms, i.e. those that appear in the expansion, from segments parsed in some other way. The segmentation is all that needs to be communicated to language services downstream of the expander, e.g. editors and pretty printers, which can pass it on to the programmer using secondary notation, e.g. colors in this document. So by examining Line 8, the programmer knows that the two instances of $x$ are spliced expressions (because they are in black), whereas the R's must be parsed in some other way, e.g. as operators of the K language (because they are in lavender). Errors in spliced terms can always be reported in terms of their original location.

(5) Segment Typing: Each spliced segment in the inferred segmentation also has a type annotation. This, together with the context independence condition, ensures that type inference at the TLM application site can be performed (by editor services or in the programmer's mind) abstractly, i.e. by reference only to the type annotations on the spliced segments, not the full expansion.

(6) Capture: Splicing is guaranteed to be capture-avoiding, so the spliced expression $x$ must refer to the binding of $x$ on Line 7 . It cannot have captured a coincidental binding of $x$ in the expansion. 
Paper Outline Sec. 2 details expression TLMs in Reason with several more case studies of varying detail, notably including TLMs for regular expressions, HTML literals, chemical structure literals and quasiquotation for Reason language terms, which can be used for implementing other TLMs. It also describes experimental integration with MetaOCaml, which can help providers reason about type correctness. Sec. 3 then briefly introduces pattern TLMs and describes the special reasoning conditions in pattern position. Sec. 4 introduces the more general parametric TLMs, which allow us to define literal notation at a type- or module-parameterized family of types. Having introduced the basic machinery by example, we proceed in Sec. 5 to describe a type-theoretic account of simple expression and pattern TLMs and formally establish the reasoning principles implied above in their essential form. The full technical details and proofs are in the accompanying technical report [Omar and Aldrich 2018]. Sec. 6 provides a brief overview of how we are implementing TLMs for Reason without modifying OCaml's type system. This implementation, called Relit, and additional implementation details, documentation and examples are available from the Relit project page:

\section{https://github.com/cyrus-/relit}

Sec. 7 compares TLMs to related work, guided by the rubric of reasoning principles just discussed. Sec. 8 concludes with a discussion of contributions, limitations and future work.

\section{EXPRESSION LITERALS}

Consider the recursive datatype Regex.t defined in Fig. 3a, which encodes regular expressions (regexes) into Reason [Thompson 1968]. Regexes are common in, for example, bioinformatics, where they are used to express patterns in DNA sequences. For example, we can construct a regex that matches the strings "A", "T", "G" or "C", which represent the four bases in DNA, as follows:

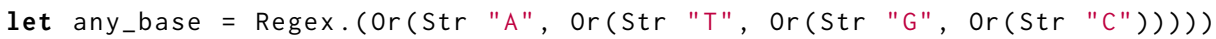

Note that in Reason, the notation Regex. (e) locally opens the module Regex within $e$, so we do not need to qualify each constructor application. Even with this shorthand, however, constructing regexes in this way is syntactically costly. Instead, we would like to have the option to use the common POSIX-style notation [IEEE 2016] when constructing values of type Regex.t, including values constructed compositionally from other regexes and strings. We solve this problem in Fig. 3b by defining a TLM named \$regex (pronounced "lit regex") that supports POSIX-style regex notation extended with splice forms for regexes (delimited by $\$($ and $)$ ) and strings (delimited by $\$ \$($ and $)$ ).

Fig. 3c shows three examples of \$regex being applied. Line 2 applies \$regex to construct the regex DNA. any_base that was described above, this time using the more concise and common POSIX regex notation. Line 3 applies \$regex again, using its regex splice form to compositionally construct a regex matching DNA sequences recognized by the BisA restriction enzyme, where the middle base can be any base. Finally, Lines 4-5 define a function, restriction_template, that constructs a more complex regex from these first two regexes and a given gene sequence represented as a string.

\subsection{Client Perspective}

Let us start from the perspective of a client programmer examining Fig. 3 but holding the underlying expansion of Fig. 3c, as well as the details of the lexer and parser, RegexLexer and RegexParser, abstract. We will return to describe the lexer and parser from the provider's perspective in Sec. 2.2.

Let us consider the second of these three TLM applications more closely:

\$regex ' (GC\$(DNA. any_base) GC)'

According to the context-free syntax of (this paper's extension to) Reason, this form is a leaf of the unexpanded parse tree, like a string literal would be. TLM names are prefixed by $\$$ to distinguish them from variables. We call the TLM argument, ' (GC\$(DNA . any_base) GC) ', a generalized literal form, following our prior work on type-specific languages [Omar et al. 2014]. The only lexical constraint 


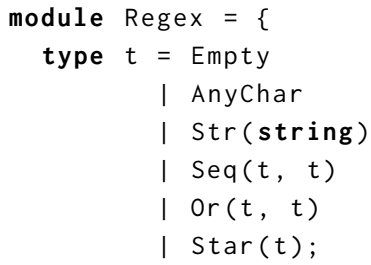

(a) The Regex module, which defines the recursive datatype Regex. $t$.

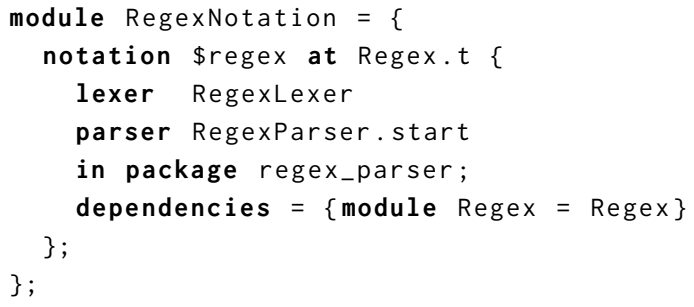

(b) The definition of \$regex. Fig. 6 defines RegexLexer and RegexParser, which are detailed in Sec. 2.2.

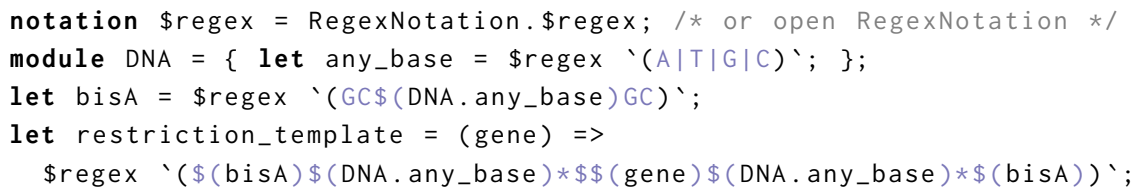

(c) Examples of the \$regex TLM being applied in a bioinformatics application.

Fig. 3. Case Study: POSIX-style regex literal notation, with support for string and regex splicing.

imposed on the literal body, i.e. the characters between ' ( and )', is that any nested occurrences of ' ( must be balanced by )', much like nested comments in Reason/OCaml. Generalized literal forms therefore lexically subsume many other literal forms. This nesting constraint is to allow TLM applications to appear inside spliced expressions. An example of nested TLM applications is shown in Fig. 5, discussed later in this section. Our prior work [Omar et al. 2014] specified other choices of outer delimitation, including layout-sensitive delimitation, but for this paper ' ( and ) ' suffice.

2.1.1 Responsibility. Responsibility for lexing, parsing and expanding each literal body is delegated uniquely to the applied TLM. TLM definitions and abbreviations (like the abbreviation on Line 1 of Fig. 3c) follow the same scoping rules as Reason modules, i.e. they can appear in modules and be accessed through module paths. When a TLM definition appears inside a module with an explicitly specified module type (a.k.a. signature), it must also appear in the module type with the same specification, up to the usual notions of type and module path equivalence in the type annotation and dependencies, which are discussed below. This is much like the situation with datatype definitions in ML. By convention, we define TLMs in a module suffixed with Notation so that client programmers can open just the relevant TLM definition(s) without bringing other definitions into scope. We will demonstrate a simple lexically scoped implicit application mechanism for situations where the same TLM is being repeatedly applied in Sec. 2.2.2.

What is fundamental about this design is that there is a well-defined protocol that follows the usual scoping rules of the language for finding the definition of the TLM uniquely responsible for each generalized literal form in a program. An editor service or documentation tool could use this protocol to integrate TLM definition lookup into a "go to definition" command. In Sec. 6, we will describe how we use an encoding of TLM definitions as modules with singleton signatures to avoid having to primitively extend OCaml.

2.1.2 Expansion Typing. Having found the definition of the \$regex TLM, the client can immediately determine the type of the expansion being generated at each application site because it is specified explicitly by the clause at Regex.t on Line 2 of Fig. 3b. The expansion type of a TLM is analogous to the return type of a function. The identity of Regex. $t$ is determined relative to the TLM definition site, not at the application site, so the module Regex need not be in scope at the application site, or it can have been shadowed by a different module. 


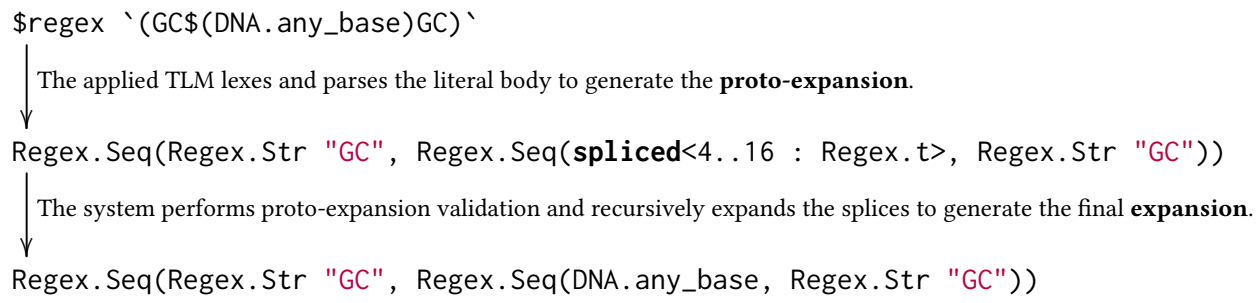

Fig. 4. TLM applications expand in two steps.

It is also worth noting here that although there is no direct mechanism for applying TLMs at the module level, this can be achieved by using OCaml's first class modules [Leroy et al. 2014].

2.1.3 Context Dependence. The system enforces a strong context independence condition on generated expansions by requiring that the TLM definition explicitly specify all modules that the expansions it generates might internally depend on. In this case, Line 6 of Fig. 3b specifies that generated expansions might use the module Regex, again as it is bound at the TLM definition site, using the module variable Regex internally. In general, the dependency can be an arbitrary module path, e.g. module List $=$ Core.Std.List. The Pervasives module is implicitly opened in each expansion. All other bindings, whether at the TLM application site or the TLM definition site, are not internally available to the expansion.

From the client's perspective, the benefit of this application site context independence discipline is clear-clients do not need to give any thought to which bindings the expansion might invisibly be assuming are in scope, as they do when using unhygienic approaches (see Sec. 7).

The benefits of making the macro definition site dependencies explicit, rather than implicitly allowing expansions to access all bindings at the definition site as in many existing macro systems, arise from the fact that this exposes the TLM's dependencies in the signature of the module where the TLM is defined. This is useful for build tools that extract dependencies by source code analysis Moreover, if an "internal" module is being used by expansions, then this will be manifest in the signature and can be corrected if this was unintended. Implicit access to the definition site would require the implementation to carefully "smuggle out" otherwise internal values to each application site, thereby skirting the abstraction discipline of ML's module system [Culpepper et al. 2005].

Enforcing this strong context independence condition is technically subtle because TLM parsers need to be able to implement splicing, i.e. they need to be able to parse terms out of the literal body for placement in the expansion. Naïvely checking that only the explicitly named dependencies are free in the expansion would inappropriately constrain application site spliced expressions, which should certainly not be prevented from referring to variables in scope at the application site. For example, consider the bottom of Fig. 4, which shows the final expansion of the example from Line 2 of Fig. 3c. In this term, both Regex and DNA are free module variables. There is nothing to distinguish references to DNA that arose from a spliced sub-expression parsed out of the literal body from those that would indicate that the context independence condition has been violated.

To address this problem, TLM parsers do not generate the final expansion directly, but rather a proto-expansion that refers to spliced terms indirectly by location relative to the start of the provided literal body. For example, the proto-expansion generated by $\$$ regex for the example above can be pretty printed as shown in the middle of Fig. 4 . Here, spliced<4..16 : Regex. $t>$ is a reference to the spliced expression DNA. any_base because the zero-indexed subsequence $[4,16)$ of GC\$(DNA. any_base)GC is DNA. any_base. We return to the type annotation on the splice reference, Regex.t, when we discuss Segment Typing in Sec. 2.1.5 below. The context independence condition can be enforced directly on the proto-expansion-the only free variable in the proto-expansion is Regex, which is an explicitly listed dependency in Fig. 3b, so all is well. 
\$html ' $(<$ div $>$

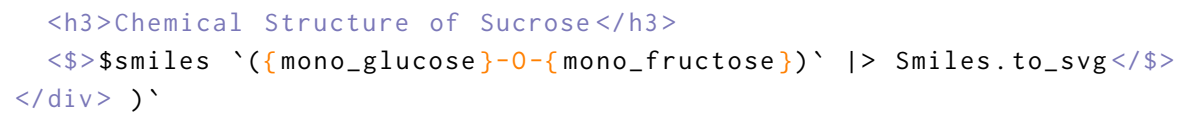

Fig. 5. A practical demonstration of nested TLM application. The colors communicate the segmentation.

2.1.4 Segmentation. The finite set of splice references in the proto-expansion generated for a literal body is called the segmentation of that literal body. The segmentation of the example above is the finite set containing one splice reference, spliced $<4 \ldots 16$ : Regex. $t>$. For the more complex example from Line 5 of Fig. 3c, the segmentation contains five splice references:

$\{$ spliced $<2 \ldots 6:$ Regex.t>, spliced $\langle 9 \ldots 21:$ Regex.t>, spliced $<26 \ldots 30:$ string $>$, spliced<33..45: Regex.t>, spliced<49.53: Regex.t>

The system checks that the segmentation does in fact segment the literal body, i.e. that the segments are in-bounds, of positive extent and non-overlapping. Adjacent spliced segments must also be separated by at least one character. The spliced segment locations can therefore be communicated unambiguously to the programmer by tools downstream of the expander, e.g. program editors and pretty printers, using secondary notation. In this paper, non-spliced segments are shown in color and spliced segments start in black.

When TLM applications are nested, a distinct color can be used at each depth. For example, Fig. 5 shows a program fragment where we transform an encoding of a chemical structure expressed using the standard SMILES notation for chemical structures [Anderson et al. 1987], extended with splicing notation, into a vector graphic, then embed this directly into a fragment of a webpage. We will detail the TLM \$html at Html.t, which implements HTML notation similar to that found in Ur/Web, in Sec. 2.2.5, and we assume a TLM \$smiles at Smiles.t, and a function, Smiles.to_svg : Smiles.t $\Rightarrow$ Html.t, not shown. In Reason, $1>$ is reverse function application.

2.1.5 Segment Typing. Each splice reference in the segmentation carries not just the location of a spliced expression but also its expected type. The identity of this type is resolved in a contextindependent manner, assuming only the dependencies explicitly specified by the TLM.

By associating a type with each spliced segment, type inference can be performed abstractly, meaning that only the segment types, together with the expansion types specified by the applied TLMs, are necessary to infer types for variables appearing in a client-side function. For example, consider the function restriction_template on Lines 4-5 of Fig. 3c. The return type of this function can be inferred to be Regex.t from the expansion type annotation on the \$regex TLM, as previously discussed. The type of the argument, gene, can be inferred to be string because the segmentation specifies the type string for the spliced segment where it appears (cf. the segmentation shown in Sec. 2.1.4 above). The context independence condition implies that gene cannot appear elsewhere in the expansion, and so no further typing constraints could possibly be collected from examining the portions of the (proto-)expansion being held abstract. Another important benefit of explicitly tracking the locations of spliced segments is that errors that originate in spliced terms can be reported in terms of their original source location [van Deursen et al. 1993].

Segment types can be communicated directly to the programmer upon request by an editor service For Reason, we plan to equip the Merlin tool [Bour et al. 2018], which is used by various Reason editor extensions (e.g. for Emacs and Vim), with a new editor command that reports the expected type of the innermost spliced segment containing the cursor. Note that because the type is explicitly stated, this information can be reported even when there is a parse or type error in a spliced expression.

Segment types are somewhat analogous to the argument types of a function. The difference is that the argument signature of a function is the same every time the function is applied, i.e. it is 
associated with the function itself, whereas the segmentation can differ for each choice of literal body. This, of course, is what gives TLMs substantially more notational flexibility, even relative to infix or mixfix function notation (where the number of subexpressions is fixed [Wieland 2009]).

2.1.6 Capture. In discussing the question of inferring a type for gene above, we neglected to consider one critical question: are we sure that the variable gene in the third spliced segment on Line 5 of Fig. $3 \mathrm{c}$ is, in fact, a reference to the argument gene of the restriction_template function? After all, if we hold the expansion abstract then it may well be that the third spliced segment appears under (i.e. captures) a different binding of the identifier gene. For more common identifiers, e.g. tmp, inadvertent capture is not difficult to imagine. For example, consider this application site:

let $\operatorname{tmp}=/ * \ldots$ application site temporary ...*/;

$\$$ html ' $(<\mathrm{h} 1><\$>f(t m p)</ \$></ h 1>)^{\prime}$;

Now consider the scenario where the proto-expansion generated by $\$ \mathrm{html}$ has the following form:

let $\operatorname{tmp}=/ * \ldots$ expansion-internal temporary ... * * ;

Html.H1Element (tmp, spliced<7..13: Html.t>);

If the final expansion was produced naïvely, by syntactically replacing the splice reference with the final expansion recursively determined for the corresponding spliced expression, then the variable tmp in the spliced expression would capture the expansion-internal binding of tmp. The result if the types of the two bindings differed would be a type error exposing the internal details of the expansion. If the types of the two bindings of tmp coincided, then there would be no static indication of the problem but there could be subtle and mysterious changes in run-time behavior.

To address this problem, splicing is guaranteed to be capture-avoiding. The final expansion is generated by recursively expanding each spliced expression and then inserting it into the final expansion via capture-avoiding substitution, which automatically alpha-varies the internal bindings of the proto-expansion as necessary. There is no need for TLM providers to manually deploy a mechanism that generates fresh variables (as in, e.g., Racket's reader macros [Flatt 2012], further discussed in Sec. 7). For example, the final expansion of the example above is alpha-equivalent to the following:

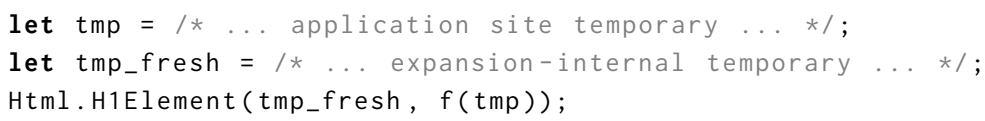

Notice that the expansion-internal binding of tmp has been alpha-varied to tmp_fresh. The reference to tmp in the spliced expression then refers, as intended, to the application site binding.

Although this strict capture avoidance discipline implies that TLMs cannot intentionally introduce bindings directly into spliced expressions, this does not imply that values cannot flow from the expansion into a spliced expression. It simply means that when this is intended, the segment type must be a function type, which serves to make this interface explicit. Reason's concise lambda notation, $(x) \Rightarrow$ e, decreases the syntactic cost of this approach. For example, we cannot define list comprehension notation like the following because the binding site of $\mathrm{x}$ is not clear:

$\$$ listcomp ' $(x+1 \mid x$ in $10 \ldots h i)$. / NO! cannot reason abstractly */

However, the following is permitted, because $x$ is bound by the spliced lambda expression and the corresponding segment type makes the type of the interface explicit:

\$listcomp ' $((\mathrm{x})=>\mathrm{x}+1 \mid \mathrm{lo} \ldots \mathrm{hi})^{\prime} / * 0 \mathrm{~K} ! * /$

Our contention is that small syntactic costs like these are more than justified by the peace of mind of knowing that unfamiliar literal notation cannot possibly be performing "magic" with the type and binding structure. We say more about the future prospect of a mechanism designed specifically for shorthand binding forms, e.g. Haskell-style do notation [Jones 2003], in Sec. 8. 


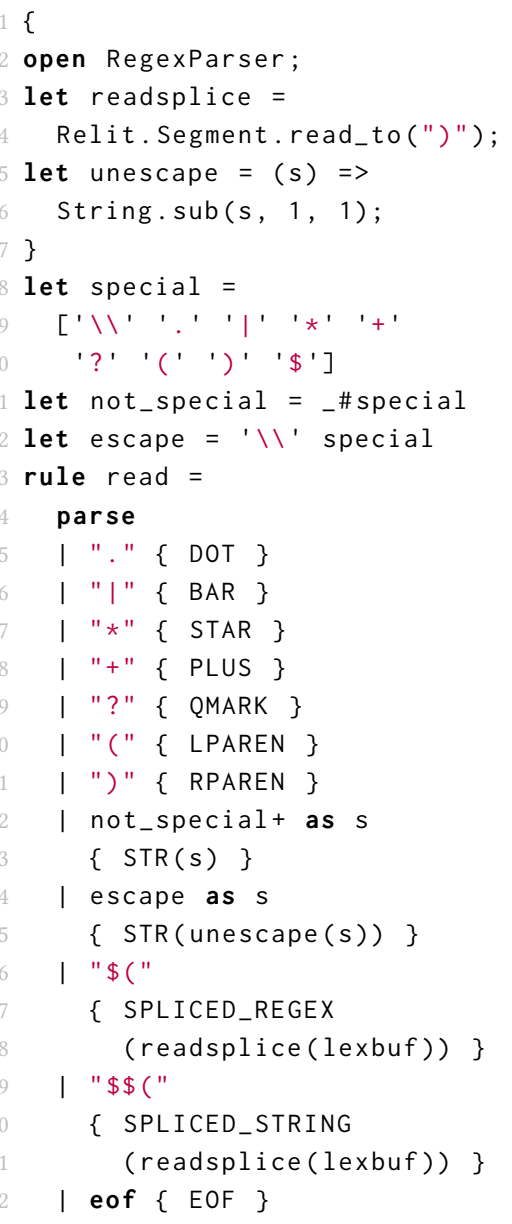

(a) RegexLexer.mll
$\%\{$

open notation Relit.\$proto_expr;

$\%\}$

\%token DOT BAR STAR PLUS QMARK LPAREN RPAREN EOF

\%token <string> STR

\%token <Relit. Segment.t> SPLICED_REGEX

\%token <Relit. Segment.t> SPLICED_STRING

$\%$ left BAR

$\%$ start <Relit.ProtoExpr.t> start

$\% \%$

start:

I e = regex; $\operatorname{EOF}\{$ e $\}$

I EOF \{'( Regex. Empty )' $\}$

regex :

I DOT \{ '( Regex. AnyChar )' \}

I $s=\operatorname{STR}\left\{\left(\operatorname{Regex} \cdot \operatorname{Str}\left(\$ \mathrm{~S}^{\prime}(\mathrm{s})^{\prime}\right)\right)^{\prime}\right\}$

| $r 1=$ regex; $r 2=$ regex

$\left.\left\{'\left(\text { Regex.Seq(' }(r 1)^{\prime},{ }^{\prime}(r 2)^{\prime}\right)\right)^{\prime}\right\}$

| $r 1=$ regex; BAR; $r 2=$ regex

$\left\{\right.$ '(Regex.Or(' $\left.\left.\left.(r 1)^{\prime}, '(r 2)^{\prime}\right)\right)^{\prime}\right\}$

$\mid r=\operatorname{regex} ; \operatorname{STAR}\left\{{ }^{\prime}\left(\operatorname{Regex} \cdot \operatorname{Star}\left({ }^{\prime}(r)^{\prime}\right)\right)^{\prime}\right\}$

$r=$ regex; PLUS

$\{$ '( let $r='(r)$;

Regex.Seq(r, Regex.Star $\left.(r)))^{\prime}\right\}$

I $r=$ regex; QMARK

$\left\{\right.$ '( Regex.Or(Regex. Empty, ' $\left.\left.\left.(r)^{\prime}\right)\right)^{\prime}\right\}$

I LPAREN; $r=$ regex; $\operatorname{RPAREN}\{r\}$

| seg = SPLICED_REGEX

$\{$ '( \$spliced '(seg: Regex.t)' )' \}

I seg = SPLICED_STRING

$\{$ '( Regex.Str

(\$spliced '(seg : string)') )' \}

(b) RegexParser.mly

Fig. 6. The lexer and parser for the \$regex TLM from Fig. 3b. See Fig. 7 for relevant definitions from Relit.

\subsection{Provider Perspective}

Let us turn now to the perspective of the TLM provider, whose principal task is to define the lexer and parser named in the TLM definition-RegexLexer and RegexParser in Fig. 3b. Our implementation of TLMs for Reason, called Relit, assumes that the provider is using ocamllex [Leroy et al. 2014] and Menhir [Pottier and Régis-Gianas 2006, 2016] to generate the lexer and parser, respectively. Menhir is a modern derivative of Yacc [Johnson 1975] with support for LR(1) grammars [Jourdan et al. 2012; Pottier and Régis-Gianas 2006]. The Reason implementation also uses these tools. Relit requires that the TLM provider package the lexer and parser into an ocamlfind package named in the TLM definition-in Fig. 3b, regex_parser-so that Relit can load them, together with their dependencies, at expansion-time. The TLM must also name the starting non-terminal-here, start on Line 4 of Fig. 3b. The ocamllex and Menhir definitions that implement RegexLexer and RegexParser appear in Fig. 6. After detailing this example in Sec. 2.2.1-2.2.2, we summarize the relevant correctness criteria in Sec. 2.2.3, describe how providers might use MetaOCaml to reason more precisely about type correctness in Sec. 2.2.4, and outline an alternative implementation strategy in Sec. 2.2 .5 that sidesteps ocamllex and Menhir to support the use of arbitrary parse functions. In particular, we implement the \$html TLM from Fig. 5 using an off-the-shelf HTML parser. 


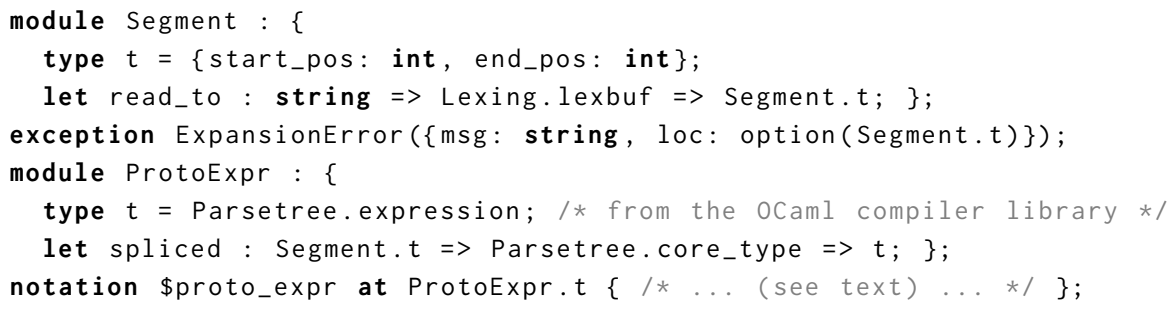

Fig. 7. A fragment of the Relit module's signature relevant to expression TLM providers.

2.2.1 RegexLexer. In most respects, the definition of the lexer in Fig. 6a is conventional. It reads various lexical patterns on Lines 8-32, emitting the corresponding tokens specified by the \%token declarations in Fig. 6b, which have been brought into scope by Line 2 of Fig. 6a. Lines 22-23 combine sequences of non-special characters (e.g. alphanumeric characters) into a single string token. Lines 24-25 implement backslash-prefixed escape sequences for characters that have special meaning in this notation for regexes, emitting a string token in each instance. ${ }^{1}$

Lines 24-31 of the lexer, which recognize the notation for regex splicing $\$(e)$, and string splicing, $\$(e)$, are more unusual. Recall from Sec. 2.1.3 that the parser must represent spliced terms abstractly, by their location within the literal body. This implies that the TLM does not need to parse the spliced expression itself, as long as it can independently decide where the spliced expression starts and ends. A paired start and end position is a segment, represented by a value of the type Segment. $t$ defined in the Relit helper library as specified on Line 2 of Fig. 7. Lines 26-28 and Lines 29-31 of the lexer produce a segment by calling a helper function, Relit.Segment.read_to, that internally invokes the Reason lexer on the provided lexing buffer until it sees an instance of the provided token outside of a Reason comment, string literal or generalized literal. If the provided token is a right delimiter also used by Reason, like ")" on Line 4 of Fig. 6a, then read_to looks for the first unmatched instance. For example, if the remaining lexing buffer contains $f("))$ ) )) A G then read_to will consume up until just before the final instance of ) and emit the appropriate segment. Similar helper functions, not shown, are available for reading out single identifiers, simple paths like X1.X2.xy and a few other unambiguously delimited fragments of the Reason syntax.

This approach has two major benefits. First, it supports splicing even within literal notations that have a very different lexical structure from Reason itself, as in this example. Second, it allows the parser to avoiding needing to link to Reason's expression grammar (though this is supported by Menhir), which substantially simplifies reasoning about ambiguities. Spliced expressions arrive into the parser as single, opaque tokens as specified on Lines 6-7 of Fig. 6b. The system will recursively expand spliced expressions after the parser has finished generating the proto-expansion.

2.2.2 RegexParser and Quasiquotation. The job of the parser is to generate a proto-expansion given the tokens generated by the lexer, or if this is not possible, to indicate an expansion error by raising either a Menhir parse error or Relit.ExpansionError (Line 4 of Fig. 7) with an appropriate error message and, if possible, an error location. In the case of expression TLMs, the proto-expansion must be a proto-expression (we consider proto-patterns in the next section). Proto-expressions are encoded as values of type Relit.ProtoExpr.t. This type is defined on Line 6 of Fig. 7 as a synonym for Parsetree.expression, which is the standard representation of expression parse trees exposed by the OCaml compiler library [Leroy et al. 2014].

In order to repurpose this existing parse tree representation for proto-expansions, we must provide some way to unambiguously represent splice references, notated earlier in the paper as

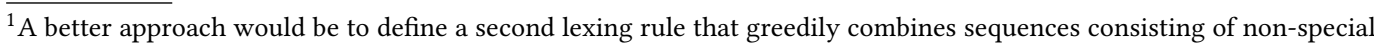
characters and escape characters into a single STR token, but for the sake of exposition, we stick to this simpler approach.
} 
spliced $<m . . n: t y>$ where m. .n specifies a segment and ty is the segment's expected type. The ProtoExpr module solves this problem by providing a function spliced that takes a segment, of type Segment.t, and a type representation, of type Parsetree.core_type, which also comes from OCaml's compiler library, and produces a corresponding value of type ProtoExpr. $t$ that uniquely represents the corresponding splice reference (see Sec. 6 for more on the internal representation)

Note that it is only because we explicitly name dependencies in TLM definitions that we can get away with using a simple, unprivileged representation of parse trees. In particular, variable renamings can be performed without needing to reason about or modify parse tree encodings in parser definitions (where variables are represented using strings, and are thus not part of the binding structure). For example, if we decided to rename the module Regex to Regexp, this renaming would need only to be performed on the module paths in the definition of $\$ r x$, as long as the name of the internal module variable in the dependencies list remains Regex.

The datatypes in OCaml's Parsetree module are necessarily intricate, given the sophistication of the OCaml system, so constructing expression encodings manually is generally too unwieldy. Addressing this class of problem is, of course, the motivation for this very paper, so it is only natural that we define TLMs for working with OCaml parse trees using familiar surface syntax, extended with splice forms. In the literature, literal notation for the host language's own parse trees is called quasiquotation, and splicing is referred to as unquotation or antiquotation [Bawden 1999; Mainland 2007; Shabalin et al. 2013]. The TLM \$proto_expr specified on Line 8 of Fig. 7 provides quasiquotation for OCaml parse trees using Reason's surface syntax. The accompanying technical report describes its implementation. We apply this TLM many times in the semantic actions in Fig. 6b. Notice, however, that the generalized literal forms in Fig. $6 \mathrm{~b}$ are not each prefixed by $\$$ proto_expr. Instead, we use the open notation directive on Line 2, which implicitly applies \$proto_expr to all unadorned generalized literal forms in its scope.

To support antiquotation, the \$proto_expr TLM repurposes TLM application and generalized literal forms, as can be observed throughout Fig. 6b. In particular, parse tree splicing is supported by unadorned generalized literal forms, e.g. on Line 23 where we implement the regex notation $r+$ in terms of sequencing and star, taking care to bind $r$ to an expansion-internal variable-also $r$ but distinguished by the segmentation-to avoid double evaluation. String splicing (which converts the spliced string expression into a parse tree of string constant form) is performed by repurposing the TLM name \$s (Line 16). There is also notation for references to spliced expressions that repurposes the TLM name \$spliced, as seen on Lines 28-32, where the regex splicing logic is implemented.

TLM-related forms are available to be repurposed in this way by $\$$ proto_expr because there is no reasonable way for \$proto_expr to support antiquotation across notational boundaries. For example, if \$proto_expr had instead used ${ }^{\wedge}(e)$ for antiquotation, then consider this example:

\$proto_expr '( let $y=\wedge(r 1) ; \$ m{ }^{\prime}\left(\ldots\left\{{ }^{\wedge}(r 2)\right\} \ldots\right)$ ' $)$ '

The sequence ${ }^{\wedge}(r 1)$ is in expression position, so antiquotation occurs. However, there is no way for \$proto_expr to know how the TLM that \$m will eventually refer to will parse the sequence ${ }^{\wedge}(r 2)$-it may end up in a spliced expression, or it may have some other meaning-so it cannot perform antiquotation. In other words, TLM expansion is fundamentally "outside-in". Notice that the segmentation does communicate the fact that $\mathrm{r} 2$ has not been antiquoted (it remains lavender), but to avoid confusion, we decided not to support the generation of TLM applications via \$proto_expr.

This issue does not come up when using TLMs to define quotation literals for languages other than Reason itself (an example is given in Sec. 3), so if this design proves too limiting, it may be reasonable to build "inside-out" quasiquotation primitively into Reason. In fact, this is already available using a preprocessor, ppx_metaquot, from the ppx_tools library [Frisch et al. 2017], or with MetaOCaml and related systems, discussed below. 
2.2.3 Correctness Criteria. In order to maintain the abstract reasoning principles discussed in Sec. 2.1, the system validates each proto-expansion generated by the parser as follows:

(1) First, the segmentation is computed and validated. This involves checking two criteria:

(a) The segments must be in-bounds, of positive extent, non-overlapping and separated.

(b) The segment types must encode valid types assuming only the TLM dependencies.

(2) Second, the proto-expansion is typechecked assuming only the TLM dependencies (plus opened Pervasives), treating each splice reference as a variable of the specified segment type.

If validation fails, the client is notified that the applied TLM is incorrectly implemented.

2.2.4 MetaOCaml. In "vanilla" OCaml, it is not possible to define a datatype that classifies only valid proto-expansions according to the correctness criteria above. The MetaOCaml type system comes closer by extending the semantics of OCaml with values of type 'a code, which are constructed using a primitive typed quasiquotation operation [Kiselyov 2014; Taha 2004]. In MetaOCaml-BER, which is an active implementation of (an extension of) MetaOCaml as a fork of the compiler, and ppx_stage, which implements a subset of MetaOCaml as an ordinary compiler plugin rather than a fork of the compiler [Dolan 2018], a value of type 'a code is represented as, and can be coerced safely to, an OCaml parse tree for the corresponding expression of type 'a [Kiselyov 2014]. It is therefore possible to implement a TLM parser using 'a code values internally, doing the coercion only at the end. However, the typing guarantee is relative to the context where the quotation was constructed, i.e. the parser's implementation, so a validation step is still needed to ensure that the assumptions are consistent with those specified by the dependencies clause.

The segmentation criteria cannot be statically enforced and, in both mentioned implementations of MetaOCaml, it is impossible to express splice references directly because they do not yet support terms with type annotations. One workaround is to define for each segment type $t$ a dummy value of type $t$ code that is replaced with a splice reference by post-processing after the coercion step.

Overall, this approach can, at least, substantially increase a TLM provider's confidence in the type correctness of the expansion logic. TLMs can be applied inside quoted code as long as the applied TLM is accessed through a dependency of the TLM being defined (so that internal dependency references correctly resolve).

2.2.5 Other Implementation Strategies. Although ocamllex and Menhir are powerful tools, they are not right for every parsing job. For example, we might want to use a different parser generator, a parser combinator library [Hutton 1992], or post-process the result of calling an existing parser to produce a corresponding proto-expansion. Fortunately, it is easy to bypass ocamllex and Menhir. Let us consider one substantial example of this approach here: the \$html TLM, shown being applied in Fig. 5, is implemented using an existing, "production grade" HTML parsing library, Markup.ml [Bachin 2018], together with a simple post-processing step as outlined in Fig. 8.

There are many ways to represent HTML data, but one simple representation is specified by $\mathrm{Html}$.t, defined in Fig. 8a. However, manually applying the constructors is tedious and, for many web programmers, the induced notation is unfamiliar. It also makes it difficult to copy-and-paste from, for example, existing HTML files, as might be useful when refactoring an existing project to use Reason. The \$html TLM defined in Fig. 8b implements the standard HTML notation extended with the tags $\langle \$\rangle$ and $\langle \$ \$\rangle$, which support Html.t splicing and string splicing, respectively. We would also in practice have splicing for HTML lists and attribute values, but we omit these here.

Fig. $8 \mathrm{c}$ shows much of the implementation of HtmlParser, which is constructed by applying a functor that constructs a module that has the same essential interface as the modules that Menhir generates, with the functions expr : string $\rightarrow$ ProtoExpr.t and pat : string $\rightarrow$ ProtoPat.t of the input module exported as the "non-terminals". The companion lexer is Relit.TrivLexer, which simply passes the whole literal body on as a single token. The implementation of expr on Line 23 


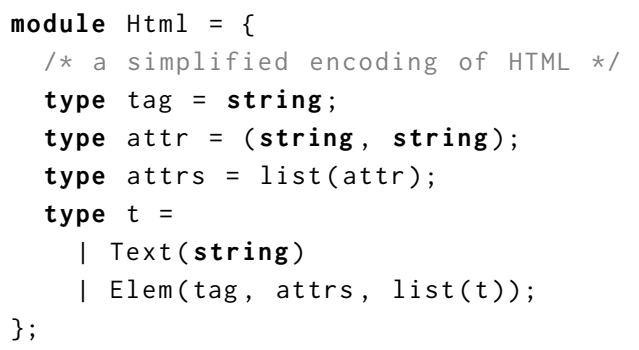

(a) The Html module, which defines Html.t.

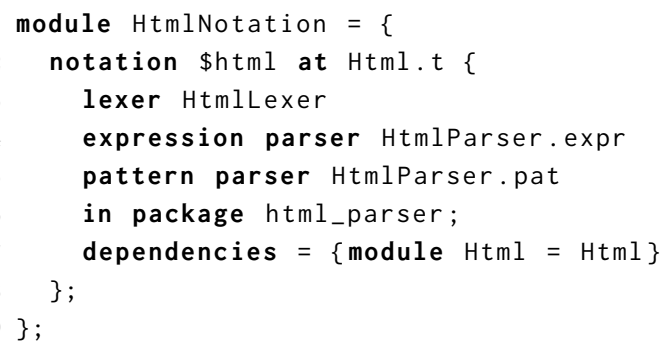

(b) The \$html expression and pattern TLMs.

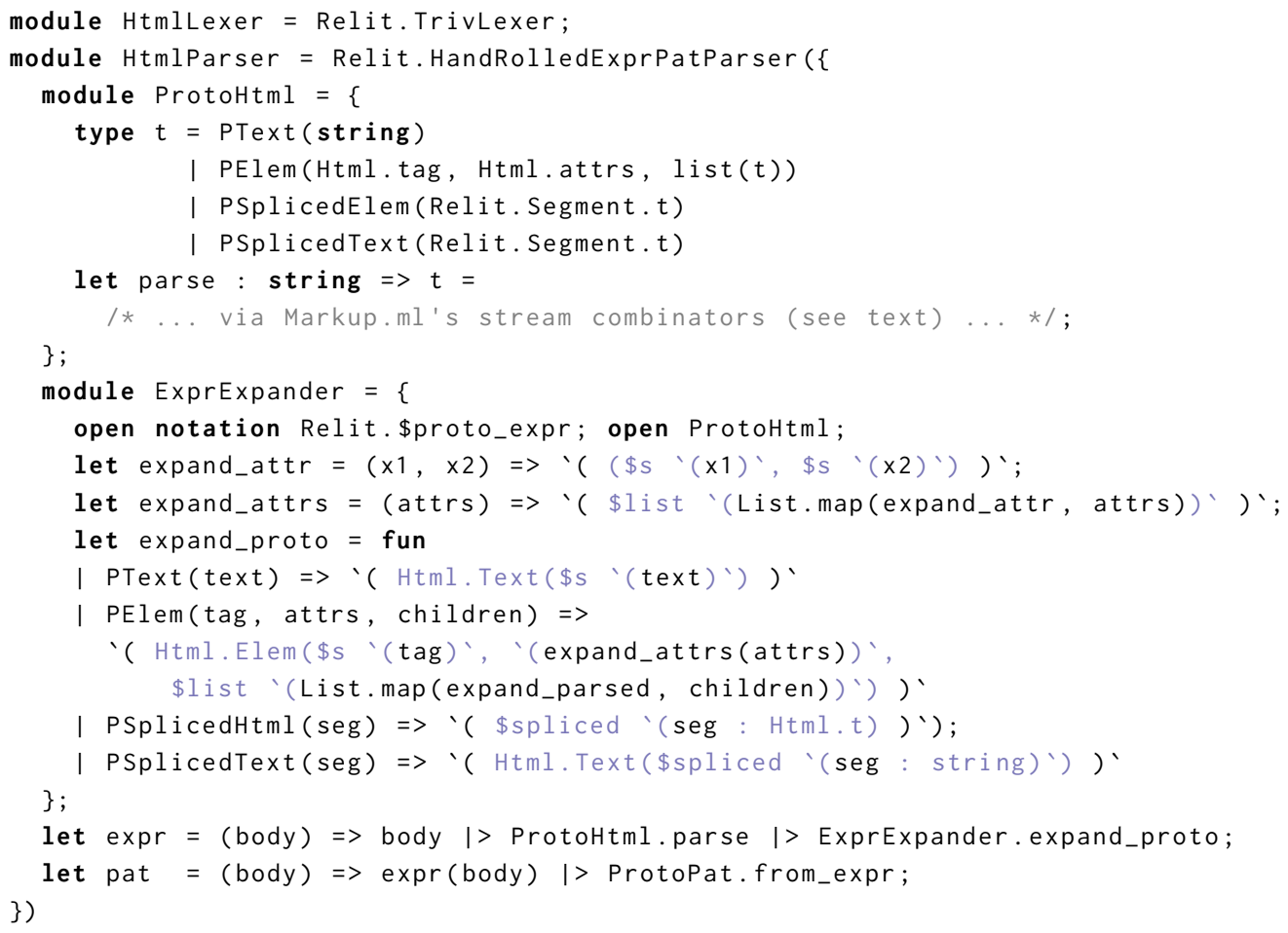

(c) The implementation of HtmlParser, which defers the parsing step to the Markup.ml library [Bachin 2018].

Fig. 8. Case Study: HTML literals as a library

of Fig. $8 \mathrm{c}$ reveals that the literal body is parsed by first calling ProtoHtml. parse, then passing the result on to ExprExpander.expand_proto. (We discuss pat in the next section.)

The ProtoHtml . parse function, elided on Lines 8-9, generates a value of the type ProtoHtml.t defined on Lines 4-7 and so named because it is similar to Html .t but distinguishes spliced segments, like a proto-expression. We omit the definition of ProtoHtml . parse but conceptually, it starts by creating a character stream from the literal body, then from that creates a Markup.ml HTML content stream, then transforms that to a stream that emits the signals from the HTML content stream until it sees a splice start tag (which is reported as a syntax error relative to the HTML standard), at which point it calls Segment.read_to, described earlier, to advance the original character stream until it recognizes the closing tag, emitting the generated segment and then returning control to the HTML content stream. Finally, it folds over this stream to produce the result of type ProtoHtml.t. 


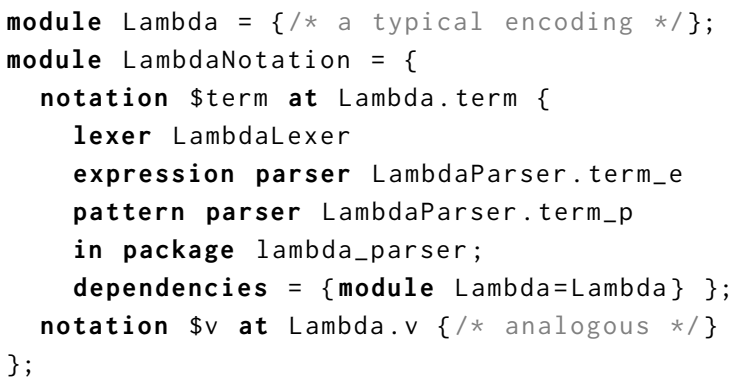

(a) Expression and pattern TLMs for Lambda terms and values

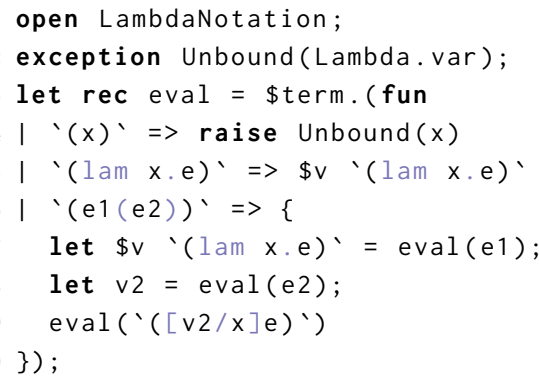

(b) A reasonably elegant Lambda evaluator

Fig. 9. Expression and pattern literal notation for lambda terms and values

The ExprExpander. expand_proto function defined, together with helper functions, on Lines 12-21 finishes the job of the TLM parser by mapping the parsed proto-HTML to a proto-expression using the \$proto_expr TLM previously described (in this case, using the \$list antiquotation form that produces a parse tree of OCaml's built in list literal form from a list of parse trees).

Reason currently builds in "JSX" literals, which also support an HTML-like notation with support for splicing. The expansion of this notation must be interpretted by a PPX rewriter. The problem is that this makes it difficult to use different HTML-related libraries at once. For example, ReasonReact and an XML library might both want to use this notation. This approach solves this problem (as well as other problems with the PPX-based approach as discussed in Sec. 7).

\section{PATTERN LITERALS}

The previous section introduced expression TLMs, which support value construction. This section introduces pattern TLMs, which support value deconstruction via the structural pattern matching facilities common to ML-like languages.

For example, the definitions outlined in Fig. 9a allow us to elegantly express an evaluator for the lambda calculus as shown in Fig. 9b. We assume that the terms of Lambda are of type Lambda. term, and the values have a different representation, of type Lambda.v, to avoid needing additional "impossible" cases. We can provide common notation for both without conflict by defining separate TLMs, \$term and \$v, respectively, in Fig. 9a. Each of these TLMs defines both expression and pattern literal notation, distinguished by qualifiers in the TLM definition as shown on Lines 5-6 of Fig. 9a, and we see examples of all four of these notations in use in Fig. 9b. These TLMs were designed to be used for operating on Lambda terms, so we chose a splicing convention inspired by the typical convention "on paper", where identifiers appearing in the literal are parsed as spliced (OCaml) variables, as indicated by the colors in Fig. 9b.

\subsection{Client Perspective}

From a client programmer's perspective, reasoning principles analogous to those described in Sec. 2.1 for expression TLMs are available. Responsibility is assigned by the same protocol, and the type annotation on the responsible TLM governs the type of the generated pattern, satisfying the Expansion Typing condition. Patterns can contain module paths and type annotations, so pattern TLMs are governed by the same Context Independence condition as expression TLMs. The same protocol around Segmentation is also enforced. The segmentation assigns a type to each spliced segment, so we can also reason about Segment Typing.

Variables in patterns do not refer to existing bindings, as in expressions, so we do not need to worry about capture avoidance. Instead, patterns introduce bindings into other expressions, e.g. the 
corresponding branch of a case analysis, so the critical question is this: given only the segmentation of a pattern literal, how can we determine exactly which variables the expansion binds? To answer, we need to preclude the possibility of "invisible bindings", so the system ensures that pattern literals bind only those variables that appear inside spliced patterns. We call this property Visibility.

Note that in OCaml, boolean guards can be associated with rules of a match expression, but the guard is not part of the syntax of patterns. (If it were, we would need to validate it as in Sec. 2.)

\subsection{Provider Perspective}

From the provider's perspective, the parser specified by the pattern parser clause differs only in that it must generate a proto-pattern, rather than a proto-expression. In Reason, this is a value of type Relit.ProtoPat.t. Much as with Relit.ProtoExpr.t from Fig. 7, this type is defined as a synonym for Parsetree.pattern from OCaml's compiler library equipped with an additional function, Relit.ProtoPat.spliced, that constructs splice references. The Visibility property is maintained by enforcing the rule that a proto-pattern simply cannot contain pattern variables.

We omit the straightforward details of the parsers in Fig. 9. In cases where the output of an expression parser uses only constructors and constants, like the HTML example in Fig. 8c, we can simply call the expression parser and then convert the result to a proto-pattern (Line 24 of Fig. 8c).

\section{PARAMETRIC TLMS}

All of the examples that we have discussed so far operate at a single specified type. This section introduces parametric TLMs, which can take type and module parameters and operate over a typeand module-parameterized family of types.

Fig. 10a shows a portion of the signature Map.S from the OCaml standard library, which specifies a polymorphic abstract data type $t$ ('a) of finite maps with keys of type key. There are many ways to implement this signature. For example, the Map. Make functor in the standard library implements this signature using balanced binary trees given a module that specifies a key type equipped with comparison functions. The Misc.StringMap module in the standard library also provides a specialized implementation of this signature with type key = string.

We can define a TLM, \$map, that is parametric over implementations of this signature, M : Map.S, and over choices of the co-domain type, a, as shown in Fig. 10b. We can then partially apply \$map to Misc. StringMap as shown on Line 1 of Fig. 10c, producing a TLM, \$stringmap, parameterized only over the type a, with expansion type Misc.StringMap.t(a). We might apply \$stringmap to a type specialize it further, e.g. \$stringmap string has expansion type Misc.StringMap.t(string) Alternatively, for TLMs where all remaining parameters are types mentioned in the expansion type, we can immediately apply the TLM to a generalized literal form as shown on Lines 2-3 of Fig. 10c. The type parameters are determined by unification using the types inferred for the spliced expressions together with the segment types specified in the segmentation, and any surrounding type constraints. In this example, we can infer a to be string because string_of_int(id) : string and the corresponding segment type is a. TLM abbreviations can themselves be parameterized to support partial application of parameters other than the last.

The proto-expansion generated by a parametric TLM can refer to its parameters. Validation checks that the proto-expansion is truly parametric-in this case, the proto-expansion must be valid for all modules $M$ : Map.S and types a. It is only after validation that we substitute the actual parameters, here Misc.StringMap for $M$ and string for a, into the final expansion.

The dependencies clause shown in previous examples can be understood in terms of parameterization over the dependencies, using the given module variables, followed immediately by partial application of the corresponding module paths. Note, however, that module parameters need an explicit signature, like functor (module function) arguments in ML. 


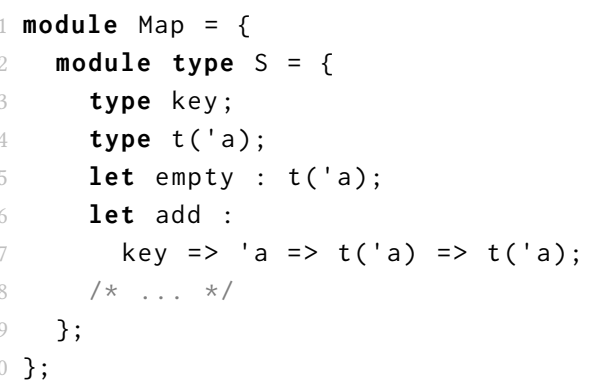

(a) The Map.S signature

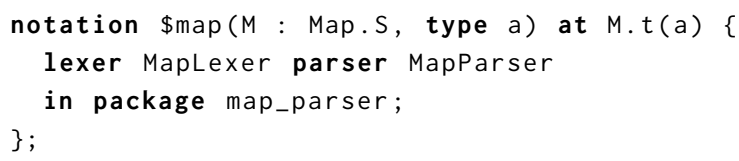

(b) The parametric TLM \$map

Fig. 10. Literal notation for all finite map implementations using parametric TLMs.

An alternative point-of-view in ML is to treat the dependencies clause as fundamental and achieve parameterization using module functions (functors), e.g. we could express \$map as follows:

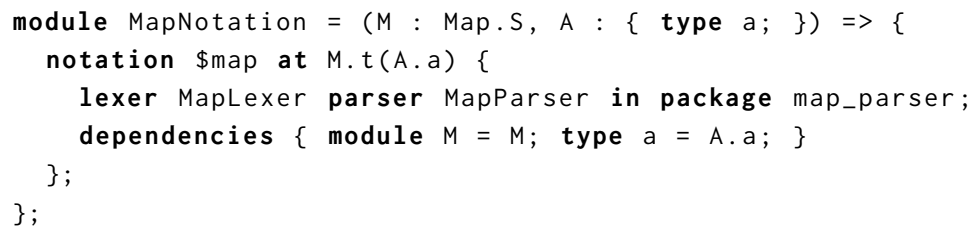

\section{TYPED LITERAL MACROS, FORMALLY}

This section presents $\mathbf{M L}^{\mathrm{Lit}}$, a calculus of simple expression and pattern TLMs based on "core ML". By the end of this section, we will have theorems that formalize the reasoning principles that were outlined informally in the previous sections. $\mathbf{M L}^{\text {Lit }}$ consists of an unexpanded language (UL) defined by typed expansion to the core language, named for the purposes of this paper $\mathbf{M L}$.

\subsection{Core Language}

Fig. 11 gives the syntax of the core language, $\mathbf{M L}$. This language forms a standard pure functional language with partial function types, quantification over types, recursive types, labeled product types, labeled sum types and support for pattern matching. ${ }^{2}$ Formally, core language terms are abstract binding trees (ABTs) identified up to alpha-equivalence, so we follow the syntactic conventions of Harper [2012]. This notational convention also clearly distinguishes core terms from unexpanded terms, which formally behave quite differently as we will describe below.

The reader is directed to PFPL [Harper 2012] for a detailed introductory account of all of these language constructs. We will only tersely summarize the static and dynamic semantics of the core language because the particularities are not critical to our ideas. The static semantics is organized around the type formation judgement, $\Delta \vdash \tau$ type, the expression typing judgement, $\Delta \Gamma \vdash e: \tau$, and the pattern typing judgement, $p: \tau \dashv \Gamma$. Type formation contexts, $\Delta$, track hypothesis of the form $t$ type, and typing contexts, $\Gamma$, track hypotheses of the form $x: \tau$. In the pattern typing judgement, $\Gamma$ collects the typing hypotheses generated by $p$. These judgements are inductively defined in the accompanying technical report along with necessary auxiliary structures and standard lemmas. The dynamic semantics of $\mathbf{M L}$ is organized around the judgements $e$ val, which says that $e$ is a value, and $e \Downarrow e^{\prime}$, which says that $e$ evaluates to the value $e^{\prime}$. As in ML, evaluation can diverge (general recursion is possible via recursive types [Harper 2012]). It can also result in match failure.

\footnotetext{
${ }^{2}$ The accompanying technical report shows how to remove pattern matching (and pattern TLMs) from the calculus by adding the usual elimination forms, e.g. unfold $(e)$ rather than the foldp $(p)$ pattern for values of recursive type.
} 


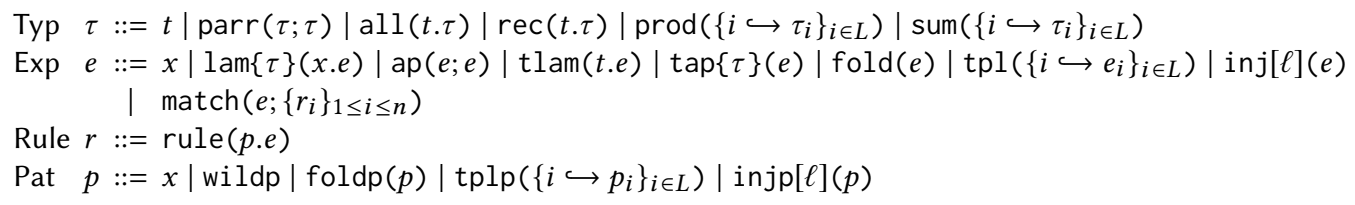

Fig. 11. Syntax of the core language, $\mathbf{M L}$, which is an entirely standard typed lambda calculus. Metavariable $x$ ranges over variables, $t$ over type variables, $\ell$ over labels and $L$ over finite sets of labels. We write $\left\{i \hookrightarrow \tau_{i}\right\}_{i \in L}$ for a finite mapping of each label $i$ in $L$ to some type $\tau_{i}$, and similarly for other sorts of terms. We write $\left\{r_{i}\right\}_{1 \leq i \leq n}$ for a finite sequence of $n>0$ rules.

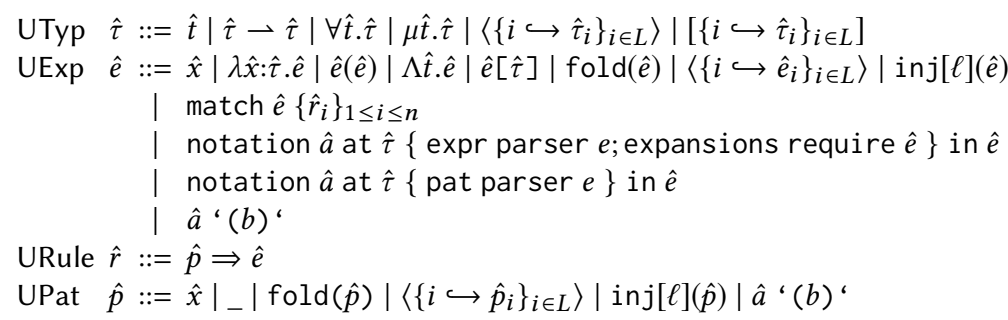

Fig. 12. Syntax of the $\mathbf{M L}^{\mathrm{Lit}}$ unexpanded language (UL). Metavariable $\hat{t}$ ranges over type identifiers, $\hat{x}$ over expression identifiers, $\hat{a}$ over TLM identifiers and $b$ over literal bodies.

\subsection{Syntax of the Unexpanded Language}

Fig. 12 defines the syntax of the unexpanded language. Unlike core language types and expressions, unexpanded expressions and types are not abstract binding trees - we do not assume the standard notions of renaming, alpha-equivalence or substitution. Instead, they are simple inductive structures. This is because unexpanded expressions remain "partially parsed" due to the presence of literal bodies, $b$, from which spliced terms might be extracted during expansion. In fact, unexpanded types and expressions do not involve variables at all, but rather identifiers, $\hat{t}$ and $\hat{x}$.

There is also a corresponding context-free textual syntax for the UL. Giving a complete definition of the context-free textual syntax with, for example, a context-free grammar is not critical to our purpose. Instead, we only posit partial metafunctions parseUTyp $(b), \operatorname{parseUExp}(b)$ and $\operatorname{parseUPat}(b)$ that go from character sequences, $b$, to unexpanded types, expressions and patterns, respectively.

\subsection{Typed Expansion}

The typed expansion judgements below specify the expansion process, which in the setting of $\mathbf{M L}^{\text {Lit }}$ occurs simultaneously with typing (see Sec. 6 for certain nuances in our implementation).

$$
\begin{array}{ll}
\hat{\Delta} \vdash \hat{\tau} \sim \tau \text { type } & \hat{\tau} \text { has well-formed expansion } \tau \\
\hat{\Delta} \hat{\Gamma} \vdash_{\hat{\Psi} ; \hat{\Phi}} \hat{e} \sim e: \tau & \hat{e} \text { has expansion } e \text { of type } \tau \\
\hat{\Delta} \vdash_{\hat{\Phi}} \hat{p} \sim p: \tau \dashv \hat{\Gamma} & \hat{p} \text { has expansion } p \text { matching } \tau
\end{array}
$$

Most of the unexpanded forms in Figure 12 mirror the expanded forms. We refer to these as the common forms. The typed expansion rules that handle common forms mirror the corresponding typing rules. The expression TLM context, $\hat{\Psi}$, and the pattern TLM context, $\hat{\Phi}$, detailed below pass through these rules opaquely. For example, the rules for variables and lambdas are shown being applied in the example derivation in Fig. 13, discussed below. The full set of rules is in the accompanying technical report [Omar and Aldrich 2018].

The only technical subtlety related to common forms has to do with the relationship between identifiers, $\hat{x}$, in the UL and variables, $x$, in the core language. We might hope to identify identifiers in the UL with variables in the core language and track the bindings in unexpanded terms using 


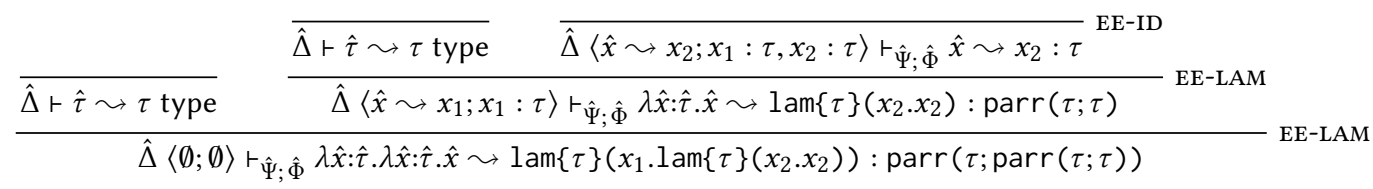

Fig. 13. An example expansion derivation demonstrating how identifiers and variables are separately tracked.

typing contexts as defined in the core language, but we cannot because the only operation for producing a new typing context from an existing typing context is context extension, written $\Gamma, x: \tau$, which is defined only when $x \notin \operatorname{dom}(\Gamma)$. When working with abstract binding trees, i.e. terms identified up to variable renaming, we typically need to give no thought to this condition because it is always possible to implicitly rename the term under consideration when shadowing occurs to discharge this requirement. However, we cannot implicitly rename unexpanded terms. Changing the definition of typing contexts would have significant implications throughout the metatheory of the core language, which we seek to avoid touching.

Instead, we define unexpanded typing contexts, $\hat{\Gamma}$, as pairs of the form $\langle\mathcal{G} ; \Gamma\rangle$, where $\mathcal{G}$ maps each expression identifier $\hat{x} \in \operatorname{dom}(\mathcal{G})$ to a variable, $x$, written $\hat{x} \sim x$. The typing context, $\Gamma$, only tracks the type of these variables. We define the identifier update operation $\mathcal{G} \uplus \hat{x} \sim x$ as mapping $\hat{x}$ to $x$, written $\hat{x} \sim x$, and deferring to $\mathcal{G}$ for all other expression identifiers, with no requirement that $\hat{x}$ be apart from $\operatorname{dom}(\mathcal{G})$. We define $\hat{\Gamma}, \hat{x} \leadsto x: \tau$ when $\hat{\Gamma}=\langle\mathcal{G} ; \Gamma\rangle$ as an abbreviation of $\langle\mathcal{G} \uplus \hat{x} \sim x ; \Gamma, x: \tau\rangle$. Unexpanded type formation contexts, $\hat{\Delta}$, are analogous.

To develop an intuition for how this formulation solves the problem, it is instructive to inspect the derivation in Fig. 13 of the expansion of the unexpanded expression $\lambda \hat{x}: \hat{\tau} . \lambda \hat{x}: \hat{\tau} . \hat{x}$ assuming $\hat{\Delta} \vdash \hat{\tau} \leadsto \tau$ type. Notice that each time Rule EE-LAM is applied, the type identifier expansion context is updated but the typing context is extended with a fresh variable, first $x_{1}$ then $x_{2}$, which is possible by alpha varying only the expansion, leaving the unexpanded term unchanged.

5.3.1 TLM Definitions. There are four unexpanded forms that are not common forms - the two TLM definition forms and the two TLM application forms. Let us start with TLM definitions. Rule EE-DEF-SETLM in Fig. 14 governs simple expression TLM (seTLM) definitions.

The first premise expands the unexpanded expansion type, $\hat{\tau}$, producing the expansion type, $\tau$.

The second premise checks that the parse function, $e_{\text {parse }}$, is a closed expanded function with input type Body and return type ParseResultE. ${ }^{3}$ The type abbreviated Body classifies encodings of literal bodies, $b$. Rather than defining Body explicitly it suffices to take as a condition that there is an isomorphism between literal bodies and values of type Body mediated in one direction by a judgement $b \downarrow_{\text {Body }} e_{\text {body }}$ that is used in the rule for TLM application discussed below. The return type, ParseResultE, abbreviates a labeled sum type, sum(Error $\hookrightarrow\langle\rangle$, SuccessE $\hookrightarrow \operatorname{PrExpr}$ ), that allows the TLM's parser to distinguish parse errors from successful parses. ${ }^{4}$

The type abbreviated PrExpr classifies encodings of proto-expressions, ̀̀ (pronounced "grave

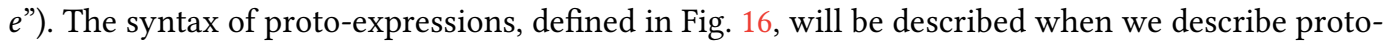
expansion validation in Sec. 5.4. As with Body, we need only take as a condition that there is an isomorphism between values of type PrExpr and closed proto-expressions, which is mediated in one direction by the proto-expression decoding judgement, $e \uparrow_{\operatorname{PrExpr}} \grave{e}$ that we will return to when we describe TLM application below (see accompanying technical report for the full conditions).

In $\mathbf{M L}^{\text {Lit }}$, we model the dependencies clause as specifying a single value dependency. The third premise of Rule EE-DEF-SETLM generates the expanded dependency, $e_{\mathrm{dep}}$ of type $\tau_{\mathrm{dep}}$, from the unexpanded dependency, $\hat{e}_{\text {dep }}$. Note that we do not need to explicitly allow for type dependencies

\footnotetext{
${ }^{3}$ Think of $e_{\text {parse }}$ as the result of parser name resolution, which produces a "compiled"-i.e. closed and expanded-term.

${ }^{4}$ In Relit, we used an exception, Relit.ExpansionError, rather than a sum type for the same purpose.
} 


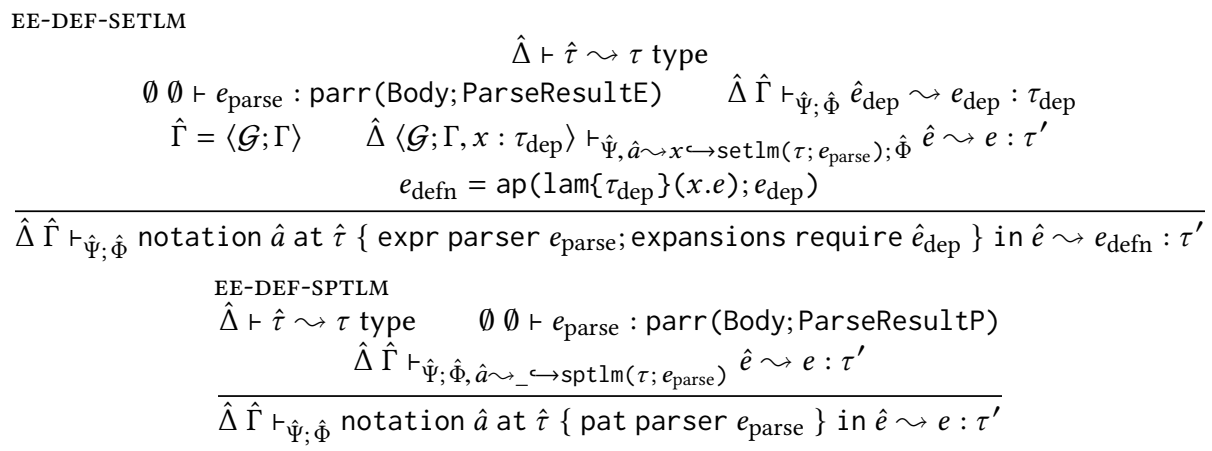

Fig. 14. The typed expansion rules for expression and pattern TLM definitions.

(which would be formally cumbersome because we cannot "tuple together" types like we can values in this setting). Instead, type dependencies can be expressed by dependency on a value of existential type to be unpacked by the expansion. This existential type can in turn be expressed in terms of universal types by the well-known encoding [Harper 2012; Reynolds 1983]. This workaround is perhaps unsurprising given that existentials relate closely to modules, which package both types and values [Harper 2012; Mitchell and Plotkin 1988].

Having processed the TLM definition, we are ready to continue into the unexpanded expression $\hat{e}$ where the TLM is bound. To activate the TLM definition for use by $\hat{e}$, the third row of premises in Rule EE-DEF-SETLM first generates a fresh variable, $x$, to stand for the value dependency. We will use this variable to instantiate the dependency in each generated expansion when we discuss TLM application below. Note that there is no corresponding expression identifier in $\mathcal{G}$. Second, the rule extends the expression TLM context, $\hat{\Psi}$, to associate the TLM identifier $\hat{a}$ with $x$, as well as with the given expansion type and parse function. If $\hat{a}$ was already defined, the previous definition is shadowed (the accompanying technical report gives some additional details on TLM contexts).

The final premise of Rule EE-DEF-SETLM, together with the conclusion of the rule, specifies the expansion of the TLM definition as being of function application form-it wraps $e$, where the variable $x$ stands free for the dependency, with a lambda binding $x$, and then immediately applies it to pass down the actual value dependency, $e_{\mathrm{dep}}$. In other words, it let-binds the dependency. This defers to the core language with regard to whether $e_{\text {dep }}$ is evaluated eagerly or lazily.

Rule EE-AP-SPTLM for pattern TLM definitions shown in Fig. 14 is analogous but simpler, because in $\mathbf{M L}^{\text {Lit }}$ patterns are entirely structural (there are no module paths that they might depend on).

5.3.2 TLM Application. The unexpanded expression form for applying an seTLM identified as $\hat{a}$

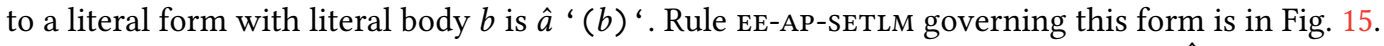

The first two premises serve simply to "look up" $\hat{a}$ in the expression TLM context, $\hat{\Psi}$, and to look up the correspondency dependency variable, $x$, in $\hat{\Gamma}$.

The third premise encodes the literal body, $e_{\mathrm{body}}$, producing a value $e_{\mathrm{body}}$ of type Body according to the body encoding judgement $b \downarrow_{\text {Body }} e_{\text {body }}$ described in Sec. 5.3.1 above.

The fourth premise applies the parse function $e_{\text {parse }}$ to the encoding of the literal body. If parsing succeeds, i.e. a value of the form inj[SuccessE] $\left(e_{\text {proto }}\right)$ results from evaluation, then $e_{\text {proto }}$ will be a value of type PrExpr (by type safety). We call $e_{\text {proto }}$ the encoding of the proto-expansion. If the parse function produces a value labeled Error, then typed expansion fails and no rule is necessary.

The fifth premise decodes the encoding of the proto-expansion using the judgement described in Sec. 5.3.1, producing the proto-expansion itself, $\dot{e}$. 


$$
\begin{aligned}
& \begin{array}{l}
\text { EE-AP-SETLM } \\
\hat{\Psi}=\hat{\Psi}^{\prime}, \hat{a} \sim x \hookrightarrow \operatorname{set} \operatorname{lm}\left(\tau ; e_{\text {parse }}\right) \quad \hat{\Gamma}=\left\langle\mathcal{G} ; \Gamma, x: \tau_{\text {dep }}\right\rangle
\end{array} \\
& b \downarrow_{\text {Body }} e_{\text {body }} \quad e_{\text {parse }}\left(e_{\text {body }}\right) \Downarrow \text { inj[SuccessE] }\left(e_{\text {proto }}\right) \quad e_{\text {proto }} \uparrow_{\operatorname{PrExpr}} \grave{e} \\
& \operatorname{seg}(\grave{e}) \text { segments } b \quad \emptyset \emptyset \vdash^{\hat{\Delta} ; \hat{\Gamma} ; \hat{\Psi} ; \hat{\Phi} ; b} \grave{e} \sim e: \operatorname{parr}\left(\tau_{\mathrm{dep}} ; \tau\right) \\
& \overline{\hat{\Delta} \hat{\Gamma} \vdash \hat{\Psi}_{;} \hat{\Phi} \hat{a}(b)^{`} \sim \operatorname{ap}(e ; x): \tau} \\
& \text { PE-AP-SPTLM } \quad \hat{\Phi}=\hat{\Phi}^{\prime}, \hat{a} \sim{ }_{-} \hookrightarrow \operatorname{spt} \operatorname{lm}\left(\tau ; e_{\text {parse }}\right) \\
& b \downarrow_{\text {Body }} e_{\text {body }} \quad e_{\text {parse }}\left(e_{\text {body }}\right) \Downarrow \overline{\text { inj }}\left[\text { SuccessP] }\left(e_{\text {proto }}\right) \quad e_{\text {proto }} \uparrow_{\text {PrPat }} \dot{p}\right. \\
& \frac{\operatorname{seg}(\hat{p}) \text { segments } b \quad \grave{p} \sim p: \tau-\|^{\hat{\Delta} ; \hat{\Phi} ; b} \hat{\Gamma}}{\hat{\Delta} \vdash_{\hat{\Phi}} \hat{a}{ }^{\prime}(b)^{`} \sim p: \tau-\| \hat{\Gamma}}
\end{aligned}
$$

Fig. 15. The typed expansion rules for expression and pattern TLM application.

The final two premises validate the proto-expansion. Proto-expansion validation is described in Sec. 5.4 below. In $\mathbf{M L}^{\text {Lit }}$, the proto-expansion does not expand directly to the final expansion but to a function, here $e$, from the dependency type, $\tau_{\text {dep }}$, to the expansion type, $\tau$, as suggested by the right-hand side of the final premise. In the conclusion of the rule, we apply the dependency variable, $x$, to produce the final expansion. ${ }^{5}$

The typed pattern expansion rule governing pattern TLM application, Rule PE-AP-SPTLM in Fig. 15, is analogous but again simpler because there is no need to pass dependencies into patterns in $\mathbf{M L}^{\mathrm{Lit}}$. We describe proto-pattern validation in Sec. 5.4 below.

\subsection{Proto-Expansion Validation}

Proto-expansion validation occurs in two steps, corresponding to the final two premises of the TLM application rules in Fig. 15.

The first of these two premises determines the segmentation of the proto-expansion by pulling out the splice references and ensures that it is valid via the predicate $\psi$ segments $b$, where $\psi$ is the segmentation. This checks that each segment has positive length and is within bounds of $b$, and that the segments do not overlap and are separated by at least one character (see accompanying technical report).

The second of these two premises typechecks the proto-expression or proto-pattern, and simultaneously generates a corresponding core language expression or pattern, from which the final expansion is constructed as described above. This step is specified by the following judgements:

$$
\begin{array}{ll}
\Delta \vdash^{\mathbb{T}} \grave{\tau} \leadsto \tau \text { type } & \grave{\tau} \text { has well-formed expansion } \tau \\
\Delta \Gamma \vdash^{\mathbb{E}} \grave{e} \sim e: \tau & \grave{e} \text { has expansion } e \text { of type } \tau \\
\grave{p} \leadsto p: \tau \|^{\mathbb{P}} \hat{\Gamma} & \grave{p} \text { has expansion } p \text { matching } \tau
\end{array}
$$

The purpose of the splicing scenes $\mathbb{T}, \mathbb{E}$ and $\mathbb{P}$ is to "remember" the contexts and literal body from the TLM application site (cf. the final premise of Rule EE-AP-SETLM in Fig. 14) for when validation encounters spliced terms. For example, expression splicing scenes, $\mathbb{E}$, are of the form $\hat{\Delta} ; \hat{\Gamma} ; \hat{\Psi} ; \hat{\Phi} ; b$.

Common Forms Most of the proto-expansion forms, including all of those elided in Fig. 16 mirror corresponding expanded forms. The rules governing proto-expansion validation for these common forms in the accompanying technical report correspondingly mirror the typing rules. Splicing scenes- $\mathbb{T}, \mathbb{E}$ and $\mathbb{P}$-pass opaquely through these rules, i.e. none of these rules can access

\footnotetext{
${ }^{5}$ In Relit, the dependency is always on the singleton module induced by the dependencies clause, which is opened immediately, so the necessary boilerplate is inserted automatically.
} 


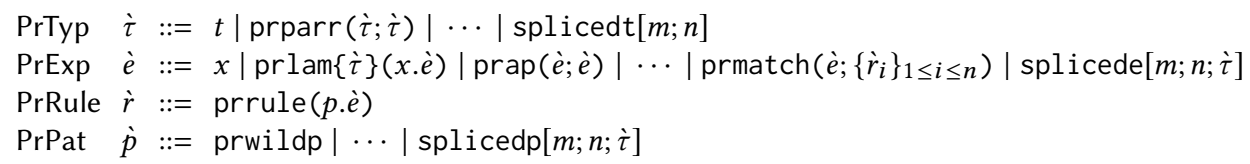

Fig. 16. Syntax of proto-expansions. Proto-expansion terms are ABTs identified up to alpha-equivalence.

the application site contexts. Notice that the initial expansion-interal typing contexts, $\Delta$ and $\Gamma$, start out empty in the rules in Fig. 15. This maintains context independence (defined formally below).

Notice that proto-rules, $\dot{r}$, involve expanded patterns, $p$, not proto-patterns, $\grave{p}$, because proto-rules appear in proto-expressions, which are generated by expression TLMs. Proto-patterns arise only from pattern TLMs. There is no variable proto-pattern form, for the reasons described in Sec. 3.

Splice References The only interesting forms in Fig. 16 are the references to spliced types, expressions and patterns. Let us consider the rule for references to spliced expressions:

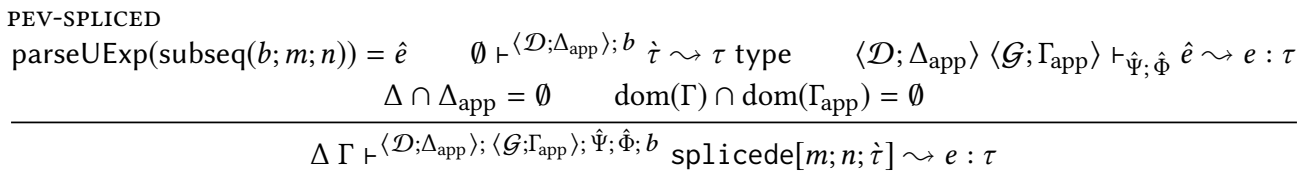

This first premise of this rule parses out the requested segment of the literal body, $b$, to produce an unexpanded expression, $\hat{e}$. The second premise performs proto-type expansion on the given type annotation, $\grave{\tau}$, producing a type, $\tau$. The expansion-internal type variables in $\Delta$ are not available to $\tau$. The third premise then invokes type expansion on $\hat{e}$ under the application site contexts, $\left\langle\mathcal{D} ; \Delta_{\text {app }}\right\rangle$ and $\left\langle\mathcal{G} ; \Gamma_{\mathrm{app}}\right\rangle$, but not the expansion-internal contexts, $\Delta$ and $\Gamma$. The final premise requires that the application site contexts are disjoint from the expansion-local type formation context. Because protoexpansions are ABTs identified up to alpha-equivalence, we can always discharge the final premise by alpha-varying the proto-expansion. This serves to enforce capture avoidance. Note that the purpose of $\mathbf{M L}^{\mathrm{Lit}}$ is to specify the necessary conditions, not to specify a particular implementation strategy. There are various ways to implement this capture avoidance condition. We will formally state the capture avoidance property in terms of capture avoiding substitution below, which is one strategy. Another is to pro-actively generate fresh variables for all internal bindings a priori.

The rule for references to spliced unexpanded types and patterns are analogous (see accompanying technical report). Note that we did not give examples of type splicing in the previous sections, but it is occasionally useful. For example, a TLM that implements a parser generator in the style of Menhir could need type splicing for reading in the non-terminal types, e.g. as on Line 9 of Fig. 6b.

\subsection{Metatheory}

Let us now sketch the main metatheoretic properties of $\mathbf{M L}^{\mathrm{Lit}}$.

5.5.1 Typed Expansion. The first property that we are interested in is simple: that typed expansion produces a well-typed expansion. As it turns out, in order to prove this theorem, we must prove the following stronger theorem, because the proto-expression validation judgement is defined mutually inductively with the typed expansion judgement (due to splicing).

Theorem 5.1 (Typed Expression Expansion (STRONG)).

(1) If $\langle\mathcal{D} ; \Delta\rangle\langle\mathcal{G} ; \Gamma\rangle \vdash_{\hat{\Psi} ; \hat{\Phi}} \hat{e} \leadsto e: \tau$ then $\Delta \Gamma \vdash e: \tau$.

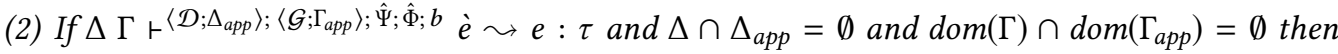
$\left(\Delta \cup \Delta_{a p p}\right)\left(\Gamma \cup \Gamma_{a p p}\right) \vdash e: \tau$. 
The additional second clause simply states that the final expansion produced by proto-expression validation is well-typed under the combined application site and expansion-internal context (because spliced terms are distinguished only in the proto-expansion, but not in the final expansion). The proof proceeds by mutual rule induction and appeal to the analogous typed pattern expansion theorem and simple lemmas about type expansion and proto-type validation. The only issue is that it is not immediately clear that the mutual induction is well-founded, because the case in the proof of part 2 for Rule PEV-SPLICED invokes part 1 of the induction hypothesis on a term that is not a sub-term of the conclusion, but rather parsed out of the literal body, $b$. To establish that the mutual induction is well-founded, then, we need to explicitly establish a decreasing metric. The intuition is that parsing a term of out a literal body cannot produce a bigger term than the term that contained that very literal body. The details are given in the accompanying technical report.

5.5.2 TLM Reasoning Principles. The following theorem summarizes the abstract reasoning principles that client programmers can rely on when applying a simple expression TLM. Informal descriptions of the labeled clauses are given inline, in gray boxes.

Theorem 5.2 (seTlM Reasoning Principles). If $\langle\mathcal{D} ; \Delta\rangle\langle\mathcal{G} ; \Gamma\rangle \vdash_{\hat{\Psi}} \hat{a}$ ' $(b)$ ' $\leadsto e: \tau$ then

1. (Expansion Typing) $\hat{\Psi}=\hat{\Psi}^{\prime}, \hat{a} \leadsto \_\hookrightarrow \operatorname{set} \operatorname{lm}\left(\tau ; e_{\text {parse }}\right)$ and $\Delta \Gamma \vdash e: \tau$

The type of the expansion is consistent with the type annotation on the applied seTLM definition.

2. (Responsibility) $b \downarrow_{\text {Body }} e_{b o d y}$ and $e_{\text {parse }}\left(e_{b o d y}\right) \Downarrow$ inj[SuccessE] $\left(e_{\text {proto }}\right)$ and $e_{\text {proto }} \uparrow_{\text {PrExpr }} \grave{e}$ The parse function of the applied TLM is responsible for generating the proto-expansion.

3. (Segmentation) $\operatorname{seg}(\grave{e})$ segments $b$

The segmentation determined by the proto-expansion segments the literal body.

4. (Segment Typing) seg $(\grave{e})=\left\{\operatorname{splicedt}\left[m_{i}^{\prime} ; n_{i}^{\prime}\right]\right\}_{0 \leq i<n_{t y}} \cup\left\{\operatorname{splicede}\left[m_{i} ; n_{i} ; \grave{i}_{i}\right]\right\}_{0 \leq i<n_{\text {exp }}}$ and

(a) $\left\{\langle\mathcal{D} ; \Delta\rangle \vdash \operatorname{parseUTyp}\left(\operatorname{subseq}\left(b ; m_{i}^{\prime} ; n_{i}^{\prime}\right)\right) \sim \tau_{i}^{\prime} \text { type }\right\}_{0 \leq i<n_{t y}}$ and $\left\{\Delta \vdash \tau_{i}^{\prime} \text { type }\right\}_{0 \leq i<n_{t y}}$

Each spliced type has a well-formed expansion.

(b) $\left\{\emptyset \vdash{ }^{\langle\mathcal{D} ; \Delta\rangle ; b} \grave{\tau}_{i} \sim \tau_{i} \text { type }\right\}_{0 \leq i<n_{\text {exp }}}$ and $\left\{\Delta \vdash \tau_{i} \text { type }\right\}_{0 \leq i<n_{\text {exp }}}$

Each segment type has a well-formed expansion.

(c) $\left\{\langle\mathcal{D} ; \Delta\rangle\langle\mathcal{G} ; \Gamma\rangle \vdash_{\hat{\Psi}} \operatorname{parseUExp}\left(\operatorname{subseq}\left(b ; m_{i} ; n_{i}\right)\right) \leadsto e_{i}: \tau_{i}\right\}_{0 \leq i<n_{\exp }}$ and $\left\{\Delta \Gamma \vdash e_{i}: \tau_{i}\right\}_{0 \leq i<n_{\exp }}$ Each spliced expression has a well-typed expansion consistent with the segment type.

5. (Capture Avoidance) $e=\left[\left\{\tau_{i}^{\prime} / t_{i}\right\}_{0 \leq i<n_{t y}},\left\{e_{i} / x_{i}\right\}_{0 \leq i<n_{\text {exp }}}\right] e^{\prime}$ for fresh variables $\left\{x_{i}\right\}_{0 \leq i<n_{\text {exp }}}$ and $\left\{t_{i}\right\}_{0 \leq i<n_{t y}}$ and some $e^{\prime}$

We can decompose the final expansion, $e$, into an "internal expression", $e^{\prime}$, with fresh variables in place of each spliced type or expression. The expansion can be produced by substituting in the expansions of these spliced types and expressions in the standard capture avoiding manner.

6. (Context Independence) $\mathrm{fv}\left(e^{\prime}\right) \subset\left\{t_{i}\right\}_{0 \leq i<n_{t y}} \cup\left\{x_{i}\right\}_{0 \leq i<n_{\exp }}$

The internal expression makes no mention of bindings in the application site context, i.e. the only free variables remaining are those standing for spliced terms.

Notice that we were able to state the hygiene properties (Capture Avoidance and Context Independence) without needing a notion of alpha-equivalence of source terms, as in typical formal accounts of hygiene [Adams 2015; Clinger and Rees 1991; Dybvig et al. 1992; Herman 2010; Herman and Wand 2008; Kohlbecker et al. 1986]. Instead, we used standard notions of capture avoiding substitution and free variables combined with the context disjointness conditions in the rules above. This is possible only because we keep track of spliced terms explicitly in the proto-expansion.

The reasoning principles theorem for pattern TLMs is below. The Visibility clause establishes that the hypotheses generated by a pattern of TLM application form are exactly the union of the hypothesis generated by the spliced patterns-there are no invisible bindings (see Sec. 3). 
Theorem 5.3 (spTlm Abstract Reasoning Principles). If $\hat{\Delta} \vdash_{\hat{\Phi}} \hat{a}$ ' $(b)^{`} \sim p: \tau \dashv \hat{\Gamma}$ where $\hat{\Delta}=\langle\mathcal{D} ; \Delta\rangle$ and $\hat{\Gamma}=\langle\mathcal{G} ; \Gamma\rangle$ then all of the following hold:

1. (Expansion Typing) $\hat{\Phi}=\hat{\Phi}^{\prime}, \hat{a} \leadsto-\hookrightarrow \operatorname{sptlm}\left(\tau ; e_{\text {parse }}\right)$ and $p: \tau-\| \Gamma$

2. (Responsibility) $b \downarrow_{\text {Body }} e_{\text {body }}$ and $e_{\text {parse }}\left(e_{\text {body }}\right) \Downarrow$ inj $\left[\right.$ SuccessP] $\left(e_{\text {proto }}\right)$ and $e_{\text {proto }} \uparrow_{\text {PrPat }} \grave{p}$

3. (Segmentation) $\operatorname{seg}(\hat{p})$ segments $b$

4. (Segment Typing) $\operatorname{seg}(\grave{p})=\left\{\operatorname{splicedt}\left[n_{i}^{\prime} ; m_{i}^{\prime}\right]\right\}_{0 \leq i<n_{t y}} \cup\left\{\operatorname{splicedp}\left[m_{i} ; n_{i} ; \grave{i}_{i}\right]\right\}_{0 \leq i<n_{p a t}}$ and

(a) $\left\{\hat{\Delta} \vdash \operatorname{parseUTyp}\left(\operatorname{subseq}\left(b ; m_{i}^{\prime} ; n_{i}^{\prime}\right)\right) \sim \tau_{i}^{\prime}\right.$ type $_{0 \leq i<n_{t y}}$ and $\left\{\Delta \vdash \tau_{i}^{\prime} \text { type }\right\}_{0 \leq i<n_{t y}}$

(b) $\left\{\emptyset \vdash^{\hat{\Delta} ; b} \grave{\tau}_{i} \leadsto \tau_{i} \text { type }\right\}_{0 \leq i<n_{\text {pat }}}$ and $\left\{\Delta \vdash \tau_{i} \text { type }\right\}_{0 \leq i<n_{\text {pat }}}$

(c) $\left\{\hat{\Delta} \vdash_{\hat{\Phi}} \operatorname{parseUPat}\left(\operatorname{subseq}\left(b ; m_{i} ; n_{i}\right)\right) \sim p_{i}: \tau_{i} \dashv\left\langle\mathcal{G}_{i} ; \Gamma_{i}\right\rangle\right\}_{0 \leq i<n_{\text {pat }}}$ and $\left\{p_{i}: \tau_{i}-\Gamma_{i}\right\}_{0 \leq i<n_{\text {pat }}}$

5. (Visibility) $\mathcal{G}=\biguplus_{0 \leq i<n_{p a t}} \mathcal{G}_{i}$ and $\Gamma=\bigcup_{0 \leq i<n_{p a t}} \Gamma_{i}$

\section{IMPLEMENTATION}

The implementation, Relit, which is an ongoing effort based on the design described in this paper, consists of a few extensions to the context-free syntax of Reason together with a parse tree pre-processor for the OCaml compiler, currently using its PPX system (discussed in Sec. 7).

TLM Definitions The Reason grammar extension turns TLM definitions into module definitions of a stereotyped form. For example, the definition of \$regex from Fig. 3b is, eliding a few minor details, implemented as shown below. The comments describe how the components correspond.

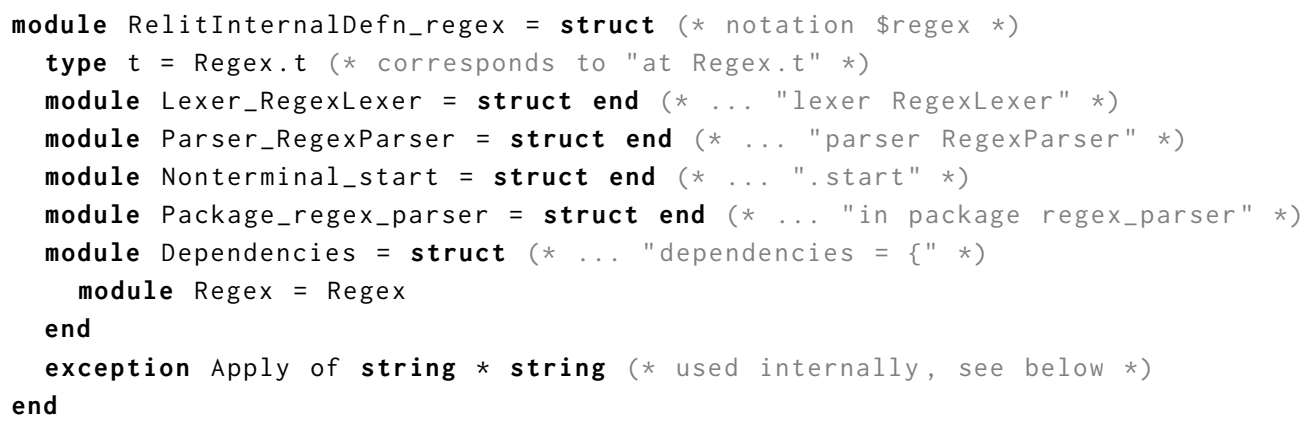

By encoding TLM definitions as modules and encoding the information from the TLM definition in module names, e.g. Lexer_RegexLexer and Nonterminal_start, we ensure that the corresponding signature is a singleton signature, i.e. it uniquely identifies the TLM definition, so TLM definitions can be included in module signatures, exported from within module definitions (including those generated by functors) and packaged using the standard tooling.

TLM Application For a TLM application, the Reason grammar extension produces an expression annotated with relit that raises the corresponding Apply exception, supplying a string containing the literal body. For example, a fragment of the example from Fig. $3 \mathrm{c}$ is below:

raise (RelitinternalDefn_regex.Apply

("You're using relit syntax without the relit ppx!", "A|T|G|C") [@relit])

The Relit preprocessor rewrites TLM applications by following the specification in the previous section, differing only in that the "dependency variable" is a "dependency path", here to Notation_regex___relit.Dependencies. The only difficult is in finding the TLM definition at all, which due to our integration into the module system may have been accessed indirectly, e.g. here via the open directive and in other situations via module synonyms, functors and so on. The trick is in the singleton restriction just described-if we can determine the signature for the module path that Apply is accessed from, we have the full definition in hand. To do so, we run the OCaml typechecker on this unexpanded representation. Because the TLM application raises an exception, 
its type can be generalized arbitrarily. As long as unrelated type errors at a particular depth are resolved (treating the TLM applications at that depth as "holes"), expansion at that depth can proceed. This occasionally causes types that incidentally appear in error messages to be more general than the programmer might expect (e.g. here restriction_template will have type ' $a=>$ ' $b$ during this "pre-typing" phase). We are working to improve error reporting and, more generally, improving integration with various other tools in the Reason / OCaml ecosystem.

Pattern TLMs are similar, but OCaml does not support exceptions in pattern position so we require a preprocessing step that turns any expression that the pattern binds over into an exception

Proto-Expansion Validation The typing phase of proto-expansion validation also defers to the OCaml typechecker, with spliced expressions represented as exceptions ascribed the corresponding segment type. The recursively generated substitutions are, after validation is complete, substituted in the standard capture-avoiding manner by simply replacing splice references with variables, and then wrapping the proto-expansion in a function that is immediately applied to the splices.

OCaml Support Although our efforts are focused on Reason, it would not be difficult to add TLM-related forms to OCaml's standard grammar, and no changes to the preprocessor just described should be needed. The only caveat is that TLMs that support splicing would then, ideally, also be agnostic to the base language grammar in use. We are exploring various ways for TLM providers to opt in to this, e.g. by defining a version of Relit.read_to from Fig. 7 for both grammars.

\section{RELATED WORK}

This section will give an overview of the many existing mechanisms that library providers might use to define new notation, and more specifically, new literal notation. Rather than evaluating these mechanisms by asking "how much syntactic power do they give the library provider?", however, our approach is to ask "what do these mechanisms quite reasonably not allow a library provider to express?", using the rubric of six reasoning principles that this paper has developed.

Syntax Definition Systems One approach available to library providers seeking to introduce new literal forms is to use a syntax definition system to extend the syntax of an existing language directly. There are hundreds of syntax definition systems of various design, and the parsers generated by these systems can be invoked to preprocess program text in various ways, e.g. by invoking them from within a build script, by using a preprocessor-aware compiler (e.g. ocamlc), or via languageintegrated preprocessing directives, e.g. the import mechanism of SugarJ [Erdweg et al. 2011], or Racket's \#lang directive [Flatt 2012].

These systems give a large amount of syntactic power to the notation provider, but this comes at a cost: with few exceptions, these systems make it difficult to reason abstractly about the six topics from Sec. 1. Rather than reiterating the points made there, let us focus on the exceptional cases.

A grammar extension system that has confronted the problem of Responsibility (but not the other problems) is Copper [Schwerdfeger 2010; Schwerdfeger and Van Wyk 2009]. Copper performs a modular grammar analysis that guarantees that determinism is conserved (i.e. ambiguities are not possible) when extensions of a certain restricted class are combined. The caveat is that the constituent extensions must prefix all newly introduced forms with marking tokens drawn from disjoint sets. To be confident that the marking tokens used are disjoint, providers must base them on, for example, the domain name system. Because the mechanism operates at the level of the context-free grammar, it is difficult for the client to define scoped abbreviations for these verbose marking tokens. TLMs can, in contrast, be abbreviated and distributed within modules following the usual scoping structure. Composition is via splicing, rather than grammar composition.

Some programming languages, notably including Rust [Rust Team 2018] and theorem provers like Agda [Norell 2007] and Coq [Coq Team 2004], support "mixfix" notation directives [Griffin 1988;

Proc. ACM Program. Lang., Vol. 2, No. ICFP, Article 106. Publication date: September 2018. 
Missura 1997; Wieland 2009]. Many of these systems enforce Capture Avoidance and applicationsite Context Independence [Danielsson and Norell 2008; Griffin 1988; Coq Team 2004; Taha and Johann 2003]. The problem is that mixfix notation requires a fixed number of sub-trees, e.g. if _ then _ else _. Rust and Coq have some ad hoc extensions for list-like literals [Coq Team 2004; Rust Team 2018]. These systems cannot express the example literal forms from this paper, because they can have any number of spliced terms.

Racket allows \#lang definitions and reader macros to gain complete control over lexing and parsing the remainder of a file [Flatt 2012]. However, this flexibility makes it difficult to reason about segmentation and even responsibility ("is a reader macro active?") Providers can optionally generate fresh variables to avoid capture, but this is not enforced. We say more about macros below.

Lorenzen and Erdweg [2013, 2016]'s SoundExt is a grammar-based syntax extension system where extension providers can equip their new forms with derived typing rules. The system then attempts to automatically verify that the expansion logic, expressed using a rewrite system, rather than an arbitrary function, is sound relative to these derived rules, so it is possible to reason about Expansion Typing. Recent work by Pombrio and Krishnamurthi [2018] instead infers the typing rules. These systems do not enforce hygiene, i.e. expansions might depend on the context and intentionally induce capture. A client can only indirectly reason about binding by inspecting the derived typing rules. There is no abstract segmentation discipline. Unlike TLMs, SoundExt supports type-dependent expansions [Lorenzen and Erdweg 2016]. The trade-off is that TLMs can generate proto-expansions, and therefore segmentations, even when the spliced expressions are ill-typed. Another important distinction is that TLMs rely on proto-expansion validation, rather than verification as in SoundExt (though providers can use MetaOCaml and related systems to reason about typing, as described in Sec. 2.2.4). The trade-off is that TLMs do not require a fully mechanized host language definition. Finally, there is no clear notion of parameterization or partial application in these systems, so it would be difficult to define notation for, for example, all finite map implementations as we demonstrated in Sec. 4.

Term Rewriting Systems Another approach - and the approach that TLMs are rooted in - is to leave the context-free syntax of the language fixed, and instead contextually rewrite existing literal forms. For example, OCaml's textual syntax now includes preprocessor extension (PPX) points used to identify terms that some external term rewriter will rewrite [Leroy et al. 2014]. For example, we could mark a string literal as follows:

[\%xml "<h1>Hello, $\{[$ first_name $]\} !</ h 1>"]$

This does help with reasoning about responsibility, but technically more than one applied preprocessor might recognize this annotation (there are, in practice, many XML/HTML libraries), and annotations do not follow scoping rules. It is impossible to reason abstractly about the other issues because the code that the preprocessor generates is unconstrained.

Term-rewriting macro systems require that the client explicitly apply the intended rewriting, implemented by a scoped macro, to the term that is to be rewritten. This addresses the issue of Responsibility more thoroughly. However, unhygienic, untyped macro systems, like the earliest variants of the Lisp macro system [Hart 1963], and, more recently, Template Haskell [Sheard and Peyton Jones 2002] and GHC's quotation system [Mainland 2007] (which is based on Template Haskell), do not allow clients to reason abstractly about the remaining issues, again because the expansion that they produce is unconstrained. There is typically some way for library providers to opt in to a capture avoidance discipline by asking for fresh variables, but this is not enforced. Note that it is not enough that with Template Haskell / GHC quotation, the generated expansion is typechecked-to satisfy the Expansion Typing criterion, it must be possible to reason abstractly about what the type of the generated expansion is. 
Hygienic term-rewriting macro systems, like those available in various Lisp-family languages [McCarthy 1978] and in Scala [Burmako 2013], prevent, or abstractly account for [Herman 2010; Herman and Wand 2008], Capture, and they enforce application-site Context Independence [Adams 2015; Clinger and Rees 1991; Dybvig et al. 1992; Kohlbecker et al. 1986]. However, a strictly hygienic term-rewriting macro system cannot be used to repurpose string literals for composite literal notation at other types because the system cannot distinguish sub-terms in the generated expansion that came from spliced segments of the literal body. From the perspective of the contextfree syntax, spliced sub-terms are neither an argument to the macro nor even a sub-term of an argument for which a tree path can be assigned [Gorn 1965; Herman 2010; Herman and Wand 2008]. Instead, they arise as the result of performing a complex operation-parsing-on an arbitrary subsequence of the string literal passed into the macro. TLMs address this problem by distinguishing spliced sub-terms explicitly in proto-expansions.

It is possible in many production-grade hygienic macro systems, e.g. in Racket [Flatt 2012], for a macro to granularly opt out of the hygiene discipline in parts of an expansion [Kiselyov 2002], but clients cannot be sure that this powerful "escape hatch" was used in a disciplined way. However, a typed literal macro system could be implemented as a layer on top of an existing hygienic macro system, e.g. in Racket, by internally deploying the escape hatch exactly as specified by this paper.

TLMs also consider the problem of definition-site context independence by specifying expansion dependencies explicitly. This sidesteps problems faced in prior attempts to integrate macros into module systems [Culpepper et al. 2005] related to "smuggling" definition site values to application sites, without violating the abstraction discipline of the module system. It also prevents issues related to cross-stage persistence, since macro definitions do not refer to definition-site values directly but rather program against the dependency signature.

Much of the research on macro systems has been for languages in the LISP tradition [McCarthy 1978] that do not have rich static type structure. The formal macro calculus studied by Herman and Wand [2008] (which is not capable of expressing new literal forms, for the reason just discussed) uses types only as a technical advice in reasoning about the binding structure of the generated expansion. The Scala macro system does support reasoning abstractly about Expansion Typing due to the return type annotations. The full calculus we have defined is the first detailed typetheoretic account of a typed, hygienic macro system of any design for an ML- or Scala-like language, i.e. one with a rich static type structure and support for pattern matching. Many of the mechanisms would be relevant even if support for parsing literal bodies was removed and replaced with term rewriting. Segmentations can be considered a refinement of the tree paths in prior work [Gorn 1965; Herman 2010]. Note also that the contributions are relevant even in dynamic languages like Racket (by simply ignoring the typing aspects, or by inserting corresponding contracts).

Research on typed staging macro systems [Davies and Pfenning 1996] like MetaML [Sheard 1999], MetaOCaml [Kiselyov 2014], MacroML [Ganz et al. 2001; Taha and Johann 2003] and Typed Template Haskell [Jones 2013] is not directly applicable to the problem of defining new literal forms because the syntax tree of the arguments cannot be inspected at all (staging macros are used mainly for optimization). Sec. 2.2.4 discussed how the typed quotations from these systems can help TLM providers reason about the type-correctness of expansions.

Some languages, including Scala [Odersky et al. 2008], build in string splicing (a.k.a. string interpolation) forms, or similar but more general fragmentary quotation forms [Slind 1991], e.g. SML/NJ [SML/NJ Documentation 2015]. These designate a particular delimiter to escape out into the expression language. The problem with using these together with macros as vehicles to introduce literal forms at various other types is 1) there is no "one-size-fits-all" escape delimiter, and 2) typing is problematic because every escaped term is checked against the same type. For example, in Fig. 1, we have splicing at two different types. 
This brings us to the most closely related work: our own prior work on type-specific languages (TSLs) [Omar et al. 2014]. Like simple expression TLMs (Sec. 2), TSLs allow library providers to programmatically control the parsing of expressions of generalized literal forms. With TSLs, parse functions are associated directly with nominal types and invoked according to a bidirectionally typed protocol. In contrast, TLMs are separately defined and explicitly applied. Accordingly, different TLMs can operate at the same type, and they can operate at any type, including structural types. In a subsequent short paper, Omar et al. [2015] suggested explicit application of simple expression TLMs also in a bidirectional typed setting [Pierce and Turner 2000], but that paper did not have any formal content. With TLMs, it is not necessary for the language to be bidirectionally typed (see Sec. 2.1.5 on ML-style type inference). The metatheory presented by Omar et al. [2014] establishes only that generated expansions are of the expected type (i.e. a variant of the Typed Expression Expansion theorem from Sec. 5.5). It does not establish the remaining abstract reasoning principles that have been the major focus of this paper. There is a context independence condition but it does not allow for any dependencies whatsoever, which is unreasonably restrictive. There is no formal hygiene theorem and indeed the formal system in the paper does not correctly handle substitution or capture avoidance, issues we emphasized because they were non-obvious in Sec. 5. Moreover, the TLM does not guarantee that a valid segmentation will exist, nor associate types with spliced segments. Finally, this prior work did not consider pattern matching, module system integration or type- or module-parameterized type families. This paper addresses all of these, resulting in a design suitable for integration into Reason/OCaml and other languages broadly in the ML tradition.

\section{DISCUSSION}

The importance of specialized notation as a "tool for thought" has long been recognized [Iverson 1980]. According to Whitehead, a good notation "relieves the brain of unnecessary work" and "sets it free to concentrate on more advanced problems" [Cajori 1928], and indeed, advances in mathematics, science and programming have often been accompanied by new notation.

Of course, this desire to "relieve the brain of unnecessary work" has motivated not only the syntax but also the semantics of languages like ML and Scala - these languages maintain a strong type and binding discipline so that programmers, and their tools, can hold certain implementation details abstract when reasoning about program behavior. In the words of Reynolds [1983], "type structure is a syntactic discipline for enforcing levels of abstraction."

Previously, these two relief mechanisms were in tension-mechanisms that allowed programmers to express new notation would obscure the type and binding structure of the program text. TLMs resolve this tension for the broad class of literal forms that generalized literal forms subsume. This class includes all of the examples enumerated in Sec. 1 (up to the choice of outermost delimiter), the varied and non-trivial case studies outlined in this paper, and the examples collected from the empirical study by Omar et al. [2014]. We anticipate many more interesting examples will emerge as the Reason community explores the mechanism.

Of course, not all possible literal notation will prove to be in good taste. The reasoning principles that TLMs provide, which are the primary contributions of this paper, allow clients to "reason around" poor literal designs, using principles analogous to those already familiar to programmers in languages like ML and Scala. Although we emphasized integration with modules, our formal system demonstrates that modules are not necessary to capture the fundamental ideas in this paper. We intend this paper to be useful to the designers of languages both near and distant from the ML tradition. More generally, we intend for the "reasoning principles first" design philosophy that we followed in this paper to be a useful example for language designers considering the merits of other "conveniences" - syntactic cost alone is not the whole story. 
Limitations Not all interesting properties of a program will, in general, be apparent from its type, particularly in a language like OCaml. As usual, programmers will sometimes need to peek behind the abstraction boundaries or rely on informal documentation to understand, in detail, what a literal notation is doing (e.g. with respect its equational properties, its side effects and so on). TLMs in languages with more expressive type systems would be commensurately more reasonable.

Notation that intentionally introduces bindings into spliced terms, like Haskell's do-notation at types equipped with monadic structure, cannot be expressed using TLMs as defined in this paper, because splicing is capture avoiding. Although we considered weakening the capture avoidance condition in various somewhat reasonable ways, e.g. by communicating which identifiers are explicitly captured by each spliced segment, it is quite difficult to communicate this structure to client programmers even with tool support. Given that do-notation is already general and comes equipped with well-understood reasoning principles, the most reasonable approach is perhaps to build it in primitively, suitably parameterized over the implementation of the MONAD signature.

Another future direction has to do with automated refactoring. The unexpanded language does not come with context-free notions of renaming and substitution. However, given a segmentation, it should be possible to "backpatch" refactorings into literal bodies. Recent work by Pombrio et al. [2017] on tracking bindings "backwards" from an expansion to the source program is likely relevant. The challenge is that the TLM's splicing logic might not be invariant to refactorings.

At several points, we allude to editor integration. However, several important questions having to do with TLM-specific syntax highlighting, incremental parsing and error recovery [Graham et al. 1979], as well as debugging [Culpepper and Felleisen 2010], remain to be considered. Another interesting direction would be to generalize TLMs to support non-textual notation in a structure editor [Omar et al. 2017a,b], thereby internalizing active code completion [Omar et al. 2012].

Concluding Remarks To conclude, let us briefly reiterate the key ideas of this paper. The focus was on ensuring that client programmers can follow simple protocols when they have questions about the syntactic structure, type structure or binding structure of a program. In answering these sorts of questions, the client is not made to look at the generated expansion itself, nor inspect the parser implementation. Instead, the programmer need only be given knowledge of the expansion type from the TLM definition and the segmentation inferred by the expander at each application site, which carries a small volume of information that can be communicated straightforwardly by standard editor services. Certain questions related to the binding structure simply do not need to be asked due to the strict context independence and capture avoidance discipline of the system.

Despite these semantic constraints, the system is able to express a number of non-trivial examples with few compromises because there is only one simple lexical constraint on literal bodies. The mechanism integrates cleanly into Reason/OCaml, with full support for its type system, module system and pattern matching system. TLM providers can use existing, mature parser generators and parse tree representations, and can opt-in to a stronger typing discipline by using various implementations of MetaOCaml. Overall, we believe that TLMs represent a distinctly reasonable and expressive new point in the design space of literal notation definition systems and macro systems.

\section{ACKNOWLEDGMENTS}

This work was supported in part by AFRL and DARPA under agreement \#FA8750-16-2-0042; by the NSA under lablet contract \#H98230-14-C-0140; and by Ravi Chugh at the University of Chicago. The authors would like to thank Robert Harper, Karl Crary and Eric Van Wyk for valuable feedback on the first author's doctoral dissertation [Omar 2017], which forms the basis of this work; Charles Chamberlain for his crucial contributions to the implementation and for valuable feedback on the paper; and members of the PL Reading Group at University of Chicago and the anonymous referees for their thoughtful critiques, which substantially improved the paper. 


\section{REFERENCES}

Michael D. Adams. 2015. Towards the Essence of Hygiene. In POPL. http://doi.acm.org/10.1145/2676726.2677013

Eric Anderson, Gilman D Veith, and David Weininger. 1987. SMILES, a line notation and computerized interpreter for chemical structures. US Environmental Protection Agency, Environmental Research Laboratory.

Anton Bachin. 2018. Markup.ml - Error-recovering streaming HTML5 and XML parsers for OCaml. http://aantron.github. io/markup.ml/. Retrieved Mar. 14, 2018.

Alan Bawden. 1999. Quasiquotation in Lisp. In Partial Evaluation and Semantic-Based Program Manipulation. http: //repository.readscheme.org/ftp/papers/pepm99/bawden.pdf

Frédéric Bour, Thomas Refis, and Gabriel Scherer. 2018. Experience report: Merlin, a Language Server for OCaml. In ICFP.

Martin Bravenboer, Eelco Dolstra, and Eelco Visser. 2007. Preventing Injection Attacks with Syntax Embeddings. In GPCE. http://doi.acm.org/10.1145/1289971.1289975

Eugene Burmako. 2013. Scala Macros: Let Our Powers Combine!: On How Rich Syntax and Static Types Work with Metaprogramming. In 4 th Workshop on Scala. Article 3, 10 pages.

Florian Cajori. 1928. A history of mathematical notations. Vol. 1. Courier Corporation.

Adam Chlipala. 2010. Ur: statically-typed metaprogramming with type-level record computation. In PLDI. http://doi.acm. org/10.1145/1806596.1806612

Adam Chlipala. 2015. Ur/Web: A Simple Model for Programming the Web. In POPL. http://dl.acm.org/citation.cfm?id=2676726 William D. Clinger and Jonathan Rees. 1991. Macros That Work. In POPL. http://doi.acm.org/10.1145/99583.99607

Ryan Culpepper and Matthias Felleisen. 2010. Debugging hygienic macros. Sci. Comput. Program. 75, 7 (2010), 496-515.

Ryan Culpepper, Scott Owens, and Matthew Flatt. 2005. Syntactic abstraction in component interfaces. In International Conference on Generative Programming and Component Engineering (GPCE).

Nils Anders Danielsson and Ulf Norell. 2008. Parsing Mixfix Operators. In 20th International Symposium on Implementation and Application of Functional Languages (IFL) - Revised Selected Papers. http://dx.doi.org/10.1007/978-3-642-24452-0_5

Rowan Davies and Frank Pfenning. 1996. A Modal Analysis of Staged Computation. In POPL.

Daniel de Rauglaudre. 2003. Camlp4 reference manual. Online (September 2003) (2003).

Stephen Dolan. 2018. Staged Metaprogramming in stock OCaml. https://github.com/stedolan/ppx_stage/. Retrieved Mar. 14, 2018.

R. Kent Dybvig, Robert Hieb, and Carl Bruggeman. 1992. Syntactic Abstraction in Scheme. Lisp and Symbolic Computation 5, 4 (1992), 295-326.

Sebastian Erdweg, Tillmann Rendel, Christian Kastner, and Klaus Ostermann. 2011. SugarJ: Library-based syntactic language extensibility. In OOPSLA.

Sebastian Erdweg and Felix Rieger. 2013. A framework for extensible languages. In GPCE.

Sebastian Erdweg, Felix Rieger, Tillmann Rendel, and Klaus Ostermann. 2012. Layout-sensitive language extensibility with SugarHaskell. In Proceedings of the 2012 Symposium on Haskell. ACM, 149-160.

Matthew Flatt. 2012. Creating Languages in Racket. Commun. ACM 55, 1 (Jan. 2012), 48-56. http://doi.acm.org/10.1145/ 2063176.2063195

Alain Frisch, Peter Zotov, and Gabriel Radanne. 2017. ppx_tools: Tools for authors of ppx rewriters. https://github.com/ ocaml-ppx/ppx_tools. Retrieved June 22, 2018.

Steven Ganz, Amr Sabry, and Walid Taha. 2001. Macros as Multi-Stage Computations: Type-Safe, Generative, Binding Macros in MacroML. In ICFP.

Saul Gorn. 1965. Explicit definitions and linguistic dominoes. In Systems and Computer Science, Proceedings of the Conference held at Univ. of Western Ontario. 77-115.

Susan L Graham, Charles B Haley, and William N Joy. 1979. Practical LR Error Recovery. Vol. 14. ACM.

T. R. G. Green. 1989. Cognitive Dimensions of Notations. In Proceedings of the HCI'89 Conference on People and Computers V (Cognitive Ergonomics). 443-460.

T.G. Griffin. 1988. Notational definition-a formal account. In Logic in Computer Science (LICS '88). 372-383.

Robert Harper. 1997. Programming in Standard ML. http://www.cs.cmu.edu/ rwh/smlbook/book.pdf. Working draft, retrieved June 21, 2015.

Robert Harper. 2012. Practical Foundations for Programming Languages. Cambridge University Press.

T. P. Hart. 1963. MACRO Definitions for LISP. Report A. I. MEMO 57. Massachusetts Institute of Technology, A.I. Lab., Cambridge, Massachusetts.

David Herman. 2010. A Theory of Typed Hygienic Macros. Ph.D. Dissertation. Northeastern University, Boston, MA.

David Herman and Mitchell Wand. 2008. A Theory of Hygienic Macros. In ESOP.

Graham Hutton. 1992. Higher-order functions for parsing. fournal of Functional Programming 2, 3 (July 1992), $323-343$. http://www.cs.nott.ac.uk/Department/Staff/gmh/parsing.ps

IEEE. 2016. Information Technology Portable Operating System Interface (POSIX) Base Specifications, Issue 7. (2016).

Proc. ACM Program. Lang., Vol. 2, No. ICFP, Article 106. Publication date: September 2018. 
Kenneth E. Iverson. 1980. Notation as a Tool of Thought. Commun. ACM 23, 8 (1980), 444-465. https://doi.org/10.1145/ 358896.358899

Stephen C Johnson. 1975. Yacc: Yet another compiler-compiler. Vol. 32. Bell Laboratories Murray Hill, NJ.

Simon Peyton Jones. 2003. Haskell 98 language and libraries: the revised report. Cambridge University Press.

Simon Peyton Jones. 2013. New directions for Template Haskell. https://ghc.haskell.org/trac/ghc/blog/Template\%20Haskell\% 20Proposal. Retrieved Mar. 14, 2018.

Jacques-Henri Jourdan, François Pottier, and Xavier Leroy. 2012. Validating LR (1) parsers. In European Symposium on Programming. Springer, 397-416.

Oleg Kiselyov. 2002. How to write seemingly unhygienic and referentially opaque macros with syntax-rules. In Scheme Workshop.

Oleg Kiselyov. 2014. The Design and Implementation of BER MetaOCaml - System Description. In Functional and Logic Programming - 12th International Symposium, FLOPS 2014, Kanazawa, Japan, fune 4-6, 2014. Proceedings. 86-102. https: //doi.org/10.1007/978-3-319-07151-0_6

Eugene E. Kohlbecker, Daniel P. Friedman, Matthias Felleisen, and Bruce Duba. 1986. Hygienic Macro Expansion. In Symposium on LISP and Functional Programming. 151-161.

Xavier Leroy, Damien Doligez, Alain Frisch, Jacques Garrigue, Didier Rémy, and Jérôme Vouillon. 2014. The OCaml system release 4.02 Documentation and user's manual. Institut National de Recherche en Informatique et en Automatique.

Florian Lorenzen and Sebastian Erdweg. 2013. Modular and automated type-soundness verification for language extensions. In ICFP. 331-342. http://dl.acm.org/citation.cfm?id=2500365

Florian Lorenzen and Sebastian Erdweg. 2016. Sound type-dependent syntactic language extension. In POPL. http: //dl.acm.org/citation.cfm?id=2837614

Geoffrey Mainland. 2007. Why it's nice to be quoted: quasiquoting for Haskell. In Haskell Workshop.

Coq Team. 2004. The Coq proof assistant reference manual. LogiCal Project. http://coq.inria.fr Version 8.0.

J. McCarthy. 1978. History of LISP. In History of programming languages I. ACM, 173-185.

Erik Meijer, Brian Beckman, and Gavin Bierman. 2006. LINQ: reconciling object, relations and XML in the .NET framework In Proceedings of the 2006 ACM SIGMOD International Conference on Management of Data. ACM.

Robin Milner, Mads Tofte, Robert Harper, and David MacQueen. 1997. The Definition of Standard ML (Revised). The MIT Press.

Stephan Albert Missura. 1997. Higher-Order Mixfix Syntax for Representing Mathematical Notation and its Parsing. Ph.D. Dissertation. ETH Zurich.

John C Mitchell and Gordon D Plotkin. 1988. Abstract types have existential type. ACM Transactions on Programming Languages and Systems (TOPLAS) 10, 3 (1988), 470-502.

U. Norell. 2007. Towards a practical programming language based on dependent type theory. PhD thesis, Chalmers University of Technology (2007).

Martin Odersky, Lex Spoon, and Bill Venners. 2008. Programming in Scala. Artima Inc.

Cyrus Omar. 2017. Reasonably Programmable Syntax. Ph.D. Dissertation. Carnegie Mellon University.

Cyrus Omar and Jonathan Aldrich. 2018. Reasonably Programmable Literal Notation: Supplemental Material. Technical Report CMU-ISR-18-104. Carnegie Mellon University.

Cyrus Omar, Darya Kurilova, Ligia Nistor, Benjamin Chung, Alex Potanin, and Jonathan Aldrich. 2014. Safely Composable Type-Specific Languages. In ECOOP.

Cyrus Omar, Ian Voysey, Michael Hilton, Jonathan Aldrich, and Matthew A. Hammer. 2017a. Hazelnut: a bidirectionally typed structure editor calculus. In POPL. http://dl.acm.org/citation.cfm?id=3009900

Cyrus Omar, Ian Voysey, Michael Hilton, Joshua Sunshine, Claire Le Goues, Jonathan Aldrich, and Matthew A. Hammer. 2017b. Toward semantic foundations for program editors. In Symposium on Advances in Programming Languages (SNAPL). http://arxiv.org/abs/1703.08694

Cyrus Omar, Chenglong Wang, and Jonathan Aldrich. 2015. Composable and Hygienic Typed Syntax Macros. In ACM Symposium on Applied Computing (SAC).

Cyrus Omar, YoungSeok Yoon, Thomas D. LaToza, and Brad A. Myers. 2012. Active Code Completion. In International Conference on Software Engineering (ICSE). http://dl.acm.org/citation.cfm?id=2337223.2337324

OWASP. 2017. OWASP Top 10 2013. https://www.owasp.org/index.php/Top_10_2017-Top_10. Retrieved May $28,2017$.

Benjamin C. Pierce and David N. Turner. 2000. Local Type Inference. ACM Trans. Program. Lang. Syst. 22, 1 (Jan. 2000), 1-44. http://doi.acm.org/10.1145/345099.345100

Justin Pombrio and Shriram Krishnamurthi. 2018. Inferring type rules for syntactic sugar. In PLDI. ACM, 812-825.

Justin Pombrio, Shriram Krishnamurthi, and Mitchell Wand. 2017. Inferring Scope through Syntactic Sugar. (2017).

François Pottier and Yann Régis-Gianas. 2006. Towards Efficient, Typed LR Parsers. Electr. Notes Theor. Comput. Sci. 148, 2 (2006), 155-180. 
François Pottier and Yann Régis-Gianas. 2016. The Menhir parser generator. http://gallium.inria.fr/fpottier/menhir. Retrieved Mar. 14, 2018. (2016).

Reason Team. 2018. Reason Guide: What and Why? https://reasonml.github.io/docs/en/what-and-why.html. Retrieved Mar. $14,2018$.

J C Reynolds. 1983. Types, abstraction and parametric polymorphism. In IFIP Congress.

Rust Team. 2018. Macros - Rust Documentation. https://doc.rust-lang.org/1.7.0/book/macros.html. Retrieved Mar. 30, $2018 .$.

August Schwerdfeger. 2010. Context-aware scanning and determinism-preserving grammar composition, in theory and practice. Ph.D. Dissertation. University of Minnesota.

August Schwerdfeger and Eric Van Wyk. 2009. Verifiable composition of deterministic grammars. In PLDI. http://doi.acm. org/10.1145/1542476.1542499

Denys Shabalin, Eugene Burmako, and Martin Odersky. 2013. Quasiquotes for Scala. Technical Report EPFL-REPORT-185242.

Tim Sheard. 1999. Using MetaML: A Staged Programming Language. Lecture Notes in Computer Science 1608 (1999).

Tim Sheard and Simon Peyton Jones. 2002. Template meta-programming for Haskell. In Haskell Workshop.

Konrad Slind. 1991. Object language embedding in Standard ML of New-Jersey. In ICFP.

SML/NJ Documentation. 2015. SML/NJ Quote/Antiquote. http://www.smlnj.org/doc/quote.html. Retrieved Nov. 3, 2015.

Walid Taha. 2004. A gentle introduction to multi-stage programming. In Domain-Specific Program Generation. Springer, $30-50$.

Walid Taha and Patricia Johann. 2003. Staged Notational Definitions. In GPCE. http://dx.doi.org/10.1007/978-3-540-39815-8_6

Ken Thompson. 1968. Programming Techniques: Regular Expression Search Algorithm. Commun. ACM 11, 6 (June 1968), 419-422. http://doi.acm.org/10.1145/363347.363387

Arie van Deursen, Paul Klint, and Frank Tip. 1993. Origin Tracking. J. Symb. Comput. (1993), 523-545. http://dx.doi.org/10. 1016/S0747-7171(06)80004-0

Arthur Whitney and Dennis Shasha. 2001. Lots O’Ticks: Real Time High Performance Time Series Queries on Billions of Trades and Quotes. SIGMOD Rec. 30, 2 (May 2001), 617-617. https://doi.org/10.1145/376284.375783

Jacob Wieland. 2009. Parsing Mixfix Expressions. Ph.D. Dissertation. Technische Universitat Berlin. 